\title{
$1 \quad$ IL-23 signaling prevents ferroptosis-driven renal immunopathology during candidiasis
}

2 Nicolas Millet ${ }^{1,2}$, Norma V. Solis ${ }^{1,2}$, Diane Aguilar², Michail S. Lionakis ${ }^{3}$, Robert T. Wheeler $^{4}$,

3 Nicholas Jendzjowsky², Marc Swidergall ${ }^{1,2,5^{*}}$

4 'Division of Infectious Diseases, Harbor-UCLA Medical Center, Torrance, CA, USA

$5{ }^{2}$ The Lundquist Institute for Biomedical Innovation at Harbor-UCLA Medical Center, Torrance, CA,

6 USA

7 Fungal Pathogenesis Section, Laboratory of Clinical Immunology and Microbiology (LCIM),

8 National Institute of Allergy and Infectious Diseases (NIAID), Bethesda, MD, USA.

$9{ }^{4}$ Department of Molecular and Biomedical Sciences, University of Maine, Orono, Maine, USA

$10{ }^{5}$ David Geffen School of Medicine at UCLA, Los Angeles, CA, USA

$11{ }^{\star}$ Correspondence: Marc Swidergall, mswidergall@lundquist.org 


\section{Abstract}

13 During infection the host relies on pattern-recognition receptors to sense invading fungal

14 pathogens to launch immune defense mechanisms. While fungal recognition and immune effector

15 responses are organ and cell type specific, during disseminated candidiasis myeloid cells exacerbate

16 collateral tissue damage. However, the complex interplay between protective antifungal immunity

17 and immunopathology remains incompletely understood. The $\beta$-glucan receptor ephrin type-A 2

18 receptor (EphA2) is required to initiate mucosal inflammatory responses during oral Candida

19 infection. Here we report that Epha2 promotes renal immunopathology during disseminated

20 candidiasis. EphA2 deficiency leads to reduced renal inflammation and injury. Comprehensive

21 analyses reveal that EphA2 limits IL-23 secretion in dendritic cells, while IL-23 signaling prevents

22 ferroptotic myeloid cell death during infection. Further, ferroptosis aggravates inflammation during

23 infection, while at the same time reducing the fungal killing capacity of macrophages. Thus, we

24 identify ferroptotic cell death as a critical pathway of Candida-mediated renal immunopathology

25 that opens a new avenue to tackle Candida infection and inflammation. 


\section{Introduction}

The first step in mounting an antifungal immune response is the recognition of extracellular pathogen-associated molecular patterns (PAMPs) of invading organism, such as Candida albicans, by various families of soluble and membrane-bound pattern recognition receptors (PRRs) ${ }^{1,2}$. Following recognition, the effective control of $C$. albicans relies on several effector mechanisms and cell types to ensure fungal clearance ${ }^{3-9}$. In contrast to mucosal candidiasis, in which IL-17-producing lymphocytes are crucial for host defense ${ }^{10-14}$, effective immunity during disseminated candidiasis relies on myeloid phagocytes ${ }^{2,15-17}$. Although myeloid phagocytes are crucial for host defense during disseminated candidiasis, their functions that are aimed to control fungal infections may also come at the cost of immunopathology ${ }^{18,19}$. In fact, excessive neutrophil accumulation in tissues late in the course of infection is deleterious in mouse models of disseminated candidiasis ${ }^{17,20}$. major target organ during disseminated candidiasis. Regulated host cell death (RCD) results in either lytic or non-lytic morphology, depending upon the signaling pathway ${ }^{22}$. Apoptosis is a nonIytic, and typically immunologically silent form of cell death ${ }^{23}$. On the other hand, lytic cell death is highly inflammatory ${ }^{23-26}$, and includes necroptosis (alternative mode of RCD mimicking features of apoptosis and necrosis ${ }^{27}$ ), pyroptosis (RCD driven by inflammasome activation ${ }^{22}$ ), and ferroptosis (iron- and lipotoxicity-dependent form of RCD ${ }^{25}$ ). Inflammatory RCD depends on the release of damage-associated molecular pattern (DAMPs) and inflammatory mediators ${ }^{28}$. RCD is increasingly understood to benefit the host ${ }^{29}$, and $C$. albicans is known to induce inflammatory RCDs, such as necroptosis, and pyroptosis to promote inflammation ${ }^{30,31}$. Indeed, deficiencies in

47 these pathways accelerate disease progression during fungal infection ${ }^{31,32}$. However, excessive 48 inflammation results in renal immunopathology during candidiasis suggesting that other 49 mechanisms or RCDs fine-tune immunopathology and fungal control. Emerging data from various 50 studies indicate an essential function of non-classical $\beta$-glucan recognition during fungal 51 infections ${ }^{33-38}$. We recently found that EphA2 acts as a $\beta$-glucan receptor in the oral cavity that 
52 triggers the production of pro-inflammatory mediators via STAT3 and MAPK on oral epithelial cells,

53 while EphA2 induces priming of neutrophil $p 47^{\text {phox }}$ to increase intracellular reactive oxygen species

54 (ROS) production to enhance killing of opsonized C. albicans yeast ${ }^{33,34}$. Although the function of this

55 novel $\beta$-glucan receptor EphA2 is well established during oral mucosal C. albicans infection ${ }^{33,34,39-41}$,

56 the role of EphA2 during disseminated candidiasis is unknown.

57 Results

\section{EphA2 deficiency increases tolerance during disseminated candidiasis}

59 Being the core of the immune response, professional immune cells act as the most

60 effective weapon to clear invading fungi. Dectin-1/CLEC7A is a major PRR of the C-type lectin

61 family, predominantly expressed on myeloid-derived cells. Classical $\beta$-glucan recognition by

62 Dectin-1 activates fungal phagocytosis and the production of pro-inflammatory cytokines ${ }^{4,42}$.

63 Consistent with previous findings ${ }^{43,44}$, Dectin-1 deficiency results in increased mortality in a

64 mouse model of disseminated candidiasis (Fig. 1A). Although EphA2 recognizes $\beta$-glucan ${ }^{33,38}$,

65 and EphA2 deficiency results in increased susceptibility to oral fungal infection ${ }^{33,34,40}$, Epha2 ${ }^{-/-}$

66 mice were more resistant during lethal C. albicans challenge (Fig. 1B, C). Since EphA2 is

67 expressed on both stromal and hematopoietic cells $33,34,37,45,46$, we generated bone marrow (BM)

68 chimeric mice and determined their resistance to disseminated candidiasis (Fig. S1).

69 Both, Epha2 ${ }^{+/+}$mice reconstituted with Epha2 $2^{-/}$BM $\quad($ knockout $\quad($ KO) $\rightarrow$ wild-type $\quad$ (WT))

70 and Epha2 $^{-/}$mice reconstituted with Epha2 ${ }^{+/+} \mathrm{BM}(\mathrm{WT} \rightarrow \mathrm{KO})$ were more resistant during HDC

71 compared to Epha2 ${ }^{+/+}$mice reconstituted with Epha2 ${ }^{+/+} \mathrm{BM}(\mathrm{WT} \rightarrow \mathrm{WT})$ (Fig. 1D). However, these

72 chimeric mice were more susceptible than Epha2 ${ }^{--}$mice reconstituted with Epha2 ${ }^{-/}$BM

$73(\mathrm{KO} \rightarrow \mathrm{KO})$, which recapitulated the phenotype observed in global Epha2 ${ }^{-/}$mice (Fig. 1B),

74 suggesting that EphA2 deficiency within cells of both, the hematopoietic and stromal

75 compartments, is required for full protection against disseminated candidiasis. 
Kidneys are a primary target organ of $C$. albicans, and invasion into the kidney medulla leads

77 to loss of renal function and death ${ }^{47,48}$. Therefore, we determined the kidney fungal burden 4 days post infection. Strikingly, no differences in renal fungal burden could be observed after 4 days of infection (Fig. 1E). We have previously shown that EphA2 activation triggers receptor-mediated invasion of oral epithelial cells ${ }^{33,40}$. The mouse model of disseminated candidiasis leads to rapid organ dissemination and clearance of $>99 \%$ of the fungus from the bloodstream within the first hour after intravenous injection ${ }^{49}$, while $C$. albicans mutants defective in invasion have reduced kidney fungal burden ${ }^{50,51}$. To rule out a possible decrease of dissemination out of the bloodstream we collected kidneys from WT and Epha2 ${ }^{-/}$mice 12 hours post infection and enumerated fungal burden. Resistance and tolerance are two complementary host defense mechanisms that increase host fitness in response to invading C. albicans ${ }^{52}$. Since WT and Epha2 ${ }^{-1-}$ mice had similar renal fungal burden (Fig. 1F), we concluded that EphA2 deficiency enhances host tolerance, independent of fungal trafficking out of the bloodstream. Although EphA2 enhances neutrophilic killing of opsonized yeast ${ }^{34}$, EphA2 has no effect on elimination of hyphae (Fig. S2), which are the dominant morphotype in kidneys after 12 hours of systemic infection (Fig. S2).

Severe renal failure plays a major role in lethality of systemic C. albicans infection ${ }^{9,53-55}$. Therefore, we determined apoptotic areas in kidneys of WT and Epha2 ${ }^{--}$mice infected C. albicans using terminal deoxynucleotidyl transferase dUTP nick end labeling (TUNEL) staining ${ }^{48}$. Although apoptosis could be detected in kidneys of both mouse strains, the overall apoptotic areas decreased in infected Epha2 ${ }^{--}$mice (Fig. 1G; Fig S3). Next, we assessed serum neutrophil gelatinaseassociated lipocalin (NGAL), a marker of acute kidney injury ${ }^{48,56}$. NGAL was strongly present in serum of WT mice, while this kidney injury marker was reduced in Epha2 ${ }^{-/}$mice (Fig. 1H). During disseminated candidiasis, both systemic inflammation as well as rapid deterioration of the infected host resembles hyper-inflammatory sepsis ${ }^{57}$. Therefore, we measured the sepsis marker soluble triggering receptor expressed on myeloid cells (TREM1) ${ }^{58}$ in serum of infected mice. TREM1 was significantly reduced in Epha2 ${ }^{--}$mice compared to WT mice (Fig. 1I). Given that EphA2 promotes 
102

103

104

105

106

107

108

109

110

111

112

113

114

115

116

117

118

119

120

121

122

123

124

125

126

127

inflammation during oral $C$. albicans infection ${ }^{33,34,39-41}$, we assessed the contribution of EphA2 in a mouse model of zymosan ( $\beta$-glucan) - induced acute kidney injury (AKI), in which immune cells and inflammation exert essential roles in kidney damage ${ }^{59,60}$. We found that EphA2 deficient mice were more resistant to AKI (Fig. 1J). Together, this data suggest that EphA2 promotes inflammation to accelerate disease progression during disseminated candidiasis.

\section{EphA2 promotes renal inflammation during disseminated candidiasis}

Although the host immune response is required to control C. albicans infections, the inflammatory response causes significant collateral tissue damage. Therefore, we determined the cytokine and chemokine response in infected kidneys in WT and Epha2 ${ }^{--}$mice. Epha2 ${ }^{--}$mice had reduced kidney levels of several pro-inflammatory cytokines, including TNFa, IL-1 $\beta$, and IL-6 (Fig. 2A). By contrast, we found that Epha2--- mice had increased levels of IL-23, IFNy, and IL-4 (Fig. 2B). Next, we assessed renal leukocyte infiltration during infection. Epha2 ${ }^{--}$mice had decreased accumulation of neutrophils and monocytes (Fig. 2C; Fig. S4), but no differences in macrophage accumulation was observed (Fig. 2C). Consistent with previous observations, EphA2 deficiency increased the accumulation of dendritic cells (DCs) during infection ${ }^{61}$ (Fig. 2D-F). These experiments suggested that EphA2 is critical to promote renal inflammation during disseminated candidiasis.

\section{EphA2 deficiency reduces renal ferroptosis during disseminated candidiasis}

To comprehensively evaluate the transcriptional response of Epha2--- mice during C. albicans infection, we performed RNA sequencing of infected kidneys. Consistent with our findings that EphA2 deficient mice had reduced renal apoptosis (Fig. 1G) and reduced inflammation (Fig. 2), KEGG pathway analysis revealed downregulation of genes involved in apoptosis and several immune response pathways (Fig. 3A). Using Gene Set Enrichment Analysis (GSEA) we found that genes involved in ferroptosis were significantly enriched in infected kidneys from WT mice compared to Epha2 ${ }^{-/}$mice (Fig. 3B). In tumor cells, SLC7A11-mediated cystine uptake promotes GPX4 protein synthesis to reduce sensitivity to ferroptotic cell death ${ }^{62,63}$. Therefore, we determined the two anti- 
ferroptotic genes SLC7a11 and GPX4, as well as LYZ2, a myeloid cell lineage marker, using RNAscope (Fig. 3C). SLC7a11 and GPX4 RNAscope particles were reduced in infected kidneys from Epha2 ${ }^{-1-}$ mice (Fig. 3D). Furthermore, GPX4 particles in myeloid cells were enriched in kidney sections from WT compared Epha2 ${ }^{-/}$mice (Fig. 3E). Using immunofluorescence, we confirmed that GPX4 protein expression was reduced in Epha2 ${ }^{-1-}$ mice in infected tissue (Fig. 3F). Ferroptosis is a lipid peroxidation-driven form of $\mathrm{RCD}^{64}$. Therefore, we determined the level of lipid peroxidation using 4-hydroxynonenal (4HNE) staining. While kidney sections of WT mice showed strong lipid peroxidation, sections of Epha2 ${ }^{-1-}$ mice had low levels of ferroptosis (Fig. 3G) suggesting that during infection host cells undergo excessive lipid peroxidation although anti-ferroptotic mechanisms are upregulated. Since $L Y Z 2^{-}$cells in kidney sections from WT and Epha2 ${ }^{--}$mice had comparable GPX4 expression, we tested whether EphA2 deficiency in macrophages $\left(L Y Z 2^{+}\right)$results in decreased ferroptosis. Using the lipid peroxidation sensor BODIPY, as well as 4HNE staining, we showed that BMDMs from Epha2 ${ }^{--}$mice underwent similar magnitudes of ferroptosis compared to WT BMDMs (Fig. S5). Collectively, this data suggest that EphA2 promotes ferroptotic cell death via an extrinsic pathway during candidiasis.

\section{Ferroptotic cell death exerts inflammation and promotes disease severity during candidiasis}

To investigate whether ferroptotic cell death exacerbates inflammation and disease progression during fungal infection, we first determined that $C$. albicans induces ferroptosis in bone marrow-derived macrophages (BMDMs) (Fig. 4A) and renal tubular epithelial cells (RTECs) (Fig. 4B). Inhibition of ferroptosis using the selective inhibitor Ferrostatin-1 (Fer-1) ${ }^{65}$ increased survival of BMDMs and RTECs during C. albicans interactions (Fig. 4C, D; Fig. S6). This finding is consistent with previous reports showing that Fer-1 treatment reduces macrophage cell death during Histoplasma capsulatum infection ${ }^{66}$. Increased BMDM survival was associated with increased C. albicans killing (Fig. 4E). Furthermore, inhibition of ferroptosis reduced cytokine secretion in BMDMs (Fig. 4F) and RTECs (Fig. 4G) suggesting that ferroptosis exerts inflammation during C. albicans infection. To analyze the contribution of ferroptotic cell death to the pathogenicity 
of disseminated candidiasis, we infected WT mice with $C$. albicans followed by daily treatment with Fer-1. Mice treated with Fer-1 were less susceptible to $C$. albicans challenge compared to vehicle control mice (Fig. 4H, I). Collectively, disseminated candidiasis induces ferroptosis in various host cell types to promote inflammation and disease progression.

\section{EphA2 and JAK signaling limit IL-23 secretion in DCs}

Following systemic C. albicans infection, DCs produce IL-23 to stimulate natural killer (NK) cell activity ${ }^{8}$. Accordingly, renal IL-23 levels correlated with increased cDC infiltration during infection in Epha2 ${ }^{--}$mice (Fig. 5A). DCs derived from Epha2 ${ }^{-1-}$ BM secreted more IL-23 when stimulated with $\beta$-glucan (Fig. 5B), while TNFa levels were unaffected (Fig. S7). Next, we examined the transcriptional response of $\beta$-glucan stimulated DCs using RNA sequencing. KEGG pathway mapping revealed downregulation of genes associated with Janus-associated kinase (JAK)- Signal transducers and activators of transcription (STAT) signaling in Epha2 ${ }^{--}$BMDCs, while genes of the peroxisome proliferator-activated receptors (PPAR) pathway were upregulated (Fig. 5C). To investigate the contribution of JAK-STAT and PPAR signaling to IL-23 secretion in DCs, we treated WT BMDCs with Ruxolitinib (JAK1/2 inhibitor), the PPARy antagonist GW9662, and the PPARy agonist Rosiglitazone followed by $\beta$-glucan stimulation. While PPARy stimulation or inhibition had no effect on IL-23 secretion, Ruxolitinib increased IL-23 levels in supernatants during $\beta$-glucan stimulation (Fig. 5D). This experiment suggested that $\beta$-glucan-induced JAKSTAT signaling reduces IL-23 secretion in DCs.

\section{IL-23 inhibits ferroptosis during disseminated candidiasis}

Besides stimulating NK cells ${ }^{8}$, IL-23 secures myeloid cell survival during candidiasis ${ }^{67}$. It is thought that this mechanism is key for maintaining sufficient numbers of phagocytes at the site of infection to ensure efficient host protection ${ }^{67}$. The link between myeloid cell survival and IL-23 was intriguing since IL-23 receptor downstream targets have been associated to counteract ferroptotic cell death ${ }^{68,69}$. To test if IL-23 prevents macrophage ferroptosis during $C$. albicans infection, we measured total cell fluorescence of oxidized C11-BODIPY. Exogenous IL-23 
reduced macrophage lipid peroxidation during C. albicans interaction (Fig. 6A). Furthermore, treatment with IL-23 increased macrophage survival (Fig. 6B), their C. albicans killing capacity (Fig. 6C), and reduced inflammation (Fig. 6C), as seen in macrophages treated with the ferroptosis inhibitor (Fig. 4). This data suggested that IL-23 signaling reduces ferroptotic macrophage cell death during $C$. albicans infection. Next, we treated Candida-infected WT mice with recombinant murine IL-23 for 3 consecutive days (Fig. 6D). IL-23 treatment increased the median survival by $>65 \%$ (6 vs. 10 days) (Fig. 6D) and reduced the renal fungal burden (Fig. 6E). We determined ferroptosis in infected kidneys by staining section for 4HNE. Treatment with rmIL-23 decreased lipid peroxidation compared to PBS control (Fig. 6G). Collectively, we show that IL-23 signaling prevents inflammatory ferroptosis in myeloid cells and improves disease outcomes during disseminated candidiasis.

\section{Discussion}

Distinct RCD mechanisms promote the resolution of infection by destroying intracellular niches which benefit the pathogen, and to coordinate an appropriate innate immune response thereafter ${ }^{23}$. However, the RCD ferroptosis has been implicated in the development of many diseases ${ }^{70-72}$. Although inflammation is required to fight infections, a reduction in myeloid cellmediated immunopathology may lead to pathogen tolerance, a phenomenon whereby the host is able to better resist infection by reducing tissue damage ${ }^{18,73}$. Our findings uncover a mechanism linking ferroptosis to immunopathology during candidiasis (Fig. S8). Here we show that myeloid and stromal cells undergo ferroptotic RCD to accelerate inflammation during fungal encounter. Macrophage ferroptosis is limited by exogenous IL-23; thus safeguarding efficient fungal clearance and controlled inflammation.

While the production of reactive oxygen species (ROS) is a key aspect of phagocytemediated host responses during $C$. albicans infection, an increase in ROS may cause lipid peroxidation and ferroptosis ${ }^{74}$. Here, we show that ferroptotic cell death benefits fungi by reducing 
the killing capacity of macrophages. Lipid peroxidation during ferroptosis results in production of 4HNE and malondialdehyde (MDA), which are able to react with primary amines on proteins or DNA to form crosslinks ${ }^{75}$. Exogenous 4HNE impairs the PKC signaling pathway in RAW macrophages ${ }^{76}$, a critical mediator of antifungal host defense ${ }^{77}$. The contribution of lipid peroxides and their degradation products to immune signaling and antifungal defense both, intrinsic and extrinsic, is being further investigated.

In response to reduced inflammatory signals, classical macrophages (M1 state) are more resistant to pro-ferroptotic treatment with a specific GPX4 inhibitor ${ }^{78}$. However, sensitization to 213 ferroptotic cell death is regulated by various mechanical stimuli ${ }^{79}$. Protrusive force is generated during phagocytosis ${ }^{80}$, and immune cells respond to mechanical forces during the polarized growth of fungal hyphae ${ }^{81}$. Likewise, fungal $\beta$-glucan recognition induces mTOR signaling in monocytes ${ }^{82}$, which has been associated with increased sensitivity to ferroptosis ${ }^{83}$. Hence,

217 mechanical forces during infection and consequently activation of distinct downstream signaling pathways sensitize myeloid cells to undergo ferroptotic cell death. conditions in recent clinical trials ${ }^{84}$. However, IL-23 has established roles during antifungal

221 immunity ${ }^{85}$. IL-23 expression is strongly induced in response to $C$. albicans via the C-type lectin 222 and TLR pathways ${ }^{86}$ and is best known for regulating IL-17 production by T cells and innate 223 lymphoid cells at epithelial barriers ${ }^{84}$. IL-23 binds to IL-12R 31 and IL-23R followed by receptor 224 complex signaling via JAK2 and Tyk2 ${ }^{85,87,88}$. In a mouse model of disseminated candidiasis, pre225 treatment with tofacitinib ${ }^{89}$ and ruxolitinib ${ }^{90}$ (JAK inhibitors) increase susceptibility and fungal 226 burden, while inflammation increases when therapy is started at the onset of disease ${ }^{90}$. 227 Furthermore, the IL-23 signaling cascade activates several STAT members, including STAT3 ${ }^{91,92 .}$ 228 In tumor cells, STAT3 inhibition induces ferroptosis via Nrf2-GPX4 signaling ${ }^{68}$, while STAT3 229 activation suppresses expression of ACSL4, an enzyme that enriches membranes with long 230 polyunsaturated fatty acids and is required for ferroptosis ${ }^{69}$. Nur et al. showed that IL-23 secures 
231 survival of myeloid cells during candidiasis by inhibiting apoptosis ${ }^{67}$. Here, we demonstrate a

232 non-canonical role of IL-23 signaling in inhibiting macrophage ferroptosis during fungal infection.

233 Historically, cell death pathways have long been considered to function in parallel with little or no

234 overlap. However, it is currently known that lytic and non-lytic RCDs, such as apoptosis,

235 necroptosis, pyroptosis, and ferroptosis are tightly connected, and can cross-regulate each other

23622 . For instance STAT3 activation (downstream of IL-23R) limits ferroptosis, pyroptosis, and

237 necroptosis ${ }^{68,93,94}$. This suggest that different types of cell death depend on the stimulant, either

238 infectious agent or drug, the cell type, and the environment, but share similar downstream signals

239 and molecular regulators.

While IL-23 inhibits ferroptotic cell death in macrophages, some cytokines, such as INFY,

241 drive ferroptosis via STAT1 signaling in tumor cells ${ }^{95}$. Whether the inflammatory tissue

242 environment during infection and their corresponding cytokines sensitize immune cells to

243 ferroptotic cell death needs to be determined. Although ferroptotic cells exhibit phosphatidylserine

244 surface exposure, which triggers removal of dying cells by macrophages ${ }^{96}$, ferroptotic cells are

245 poorly engulfed and cleared ${ }^{97}$. Thus, we speculate that inefficient clearance of ferroptotic immune

246 cells might accelerate immunopathology during infection.

In DCs $\beta$-glucan recognition induces several signaling pathways, including AKT, MAPKs,

$248 \mathrm{IKK}$, and NF-KB ${ }^{86}$. Here we show that activation of the $\beta$-glucan receptor EphA2 represses IL-23

249 secretion suggesting that $\beta$-glucan recognition stimulates IL-23 expression via Dectin-1/TLR-2 ${ }^{98}$,

250 but limits the cytokine secretion via non-classical $\beta$-glucan recognition. EphA2 activates

251 JAK1/STAT3 signaling ${ }^{99}$, and STAT3 deficient DCs exhibit increased IL-23 production after

252 stimulation ${ }^{100}$. Accordingly, EphA2 deficient DCs and inhibition of JAK signaling in $\beta$-glucan

253 stimulated DCs increase IL-23 secretion. Taken together, EphA2-JAK-STAT signaling negatively

254 regulates an inflammatory IL-23 DC phenotype during fungal encounter

255 Collectively, our study demonstrates that ferroptotic host cell death is linked to

256 immunopathology and can be targeted by recombinant cytokine therapy during fungal infection. 
bioRxiv preprint doi: https://doi.org/10.1101/2021.12.19.473386; this version posted December 21, 2021. The copyright holder for this preprint (which was not certified by peer review) is the author/funder, who has granted bioRxiv a license to display the preprint in perpetuity. It is made available under aCC-BY-NC-ND 4.0 International license.

257 We postulate that strategies to inhibit ferroptotic cell death during infection will have important

258 therapeutic benefits. 


\section{Methods}

260

261

262

Ethics statement. All animal work was approved by the Institutional Animal Care and Use Committee (IACUC) of the Lundquist Institute at Harbor-UCLA Medical Center.

Subject details. For in vivo animal studies, age-and sex matched mice were used. Animals were bred/housed under pathogen-free conditions at the Lundquist Institute. Animals were randomly assigned to the different treatment groups. Researchers were not blinded to the experimental groups because the endpoints (survival, fungal burden, cytokine levels) were objective measures of disease severity. Epha2 ${ }^{-1-}$ (B6-Epha2 ${ }^{\text {tm1Jrui} / J) ~ m i c e ~ w e r e ~ p r o v i d e d ~ b y ~ A . ~ W a y n e ~ O r r ~}{ }^{46}$. C57BL/6 control and $\mathrm{Clec7a}^{-1-}$ mice were purchased from The Jackson Laboratory. All mice were cohoused for at least 1 week before the experiments.

Mouse model of HDC. Resistance to disseminated candidiasis was tested in the mouse model of HDC using 6- to 8-week-old mice (C57BL/6J background) as previously described ${ }^{17}$. The C. albicans SC5314 strain was serially passaged 3 times in YPD broth, grown at $30^{\circ} \mathrm{C}$ at $200 \mathrm{rpm}$ for 16-24 hours at each passage. Yeast cells were washed, and $2.5 \times 10^{5}$ or $1.25 \times 10^{5} \mathrm{C}$. albicans cells injected intravenously via the lateral tail vein. For survival experiments, mice were monitored three times daily and moribund mice were humanely euthanized. To determine organ fungal burden, mice were sacrificed after 12 hours and 4 days of infection, after which the kidneys were harvested, weighed, homogenized, and quantified on sabouraud dextrose agar plates containing $80 \mathrm{mg} / \mathrm{L}$ chloramphenicol. For histology, mouse kidneys were fixed in $10 \%$ buffered formalin and embedded in paraffin.

To inhibited ferroptosis during infection mice were treated daily intraperitoneally (start 6 hours post infection) with $10 \mathrm{mg} / \mathrm{kg}$ Ferrostatin-1 (SelleckChem; >99\% purity) dissolved in $0.9 \%$ $\mathrm{NaCl}$. In another experiment mice were treated intravenously 2 days post infection with $12.5 \mu \mathrm{g} / \mathrm{kg}$ recombinant murine IL-23 (1887-ML-010/CF, R\&D Systems) for 3 consecutive days.

Immunohistochemistry. Apoptotic cell death was determined as previously described ${ }^{48}$. Briefly, terminal deoxynucleotidyl transferase nick end labeling (TUNEL) staining was performed using 
the in situ apoptosis detection kit (ApopTag, S7100, Chemicon, Temecula, CA, USA) according to the manufacturer's protocol with minor modifications. The paraffin-embedded renal sections were placed on poly-L-lysine coated glass slides, deparaffinized in xylene and rehydrated in a graded series of alcohol. Then treated with protease $\mathrm{K}(20 \mathrm{~g} / \mathrm{ml})$ for $15 \mathrm{~min}$ at room temperature. Sections were incubated with reaction buffer containing terminal deoxynucleotidyltransferase at $37^{\circ} \mathrm{C}$ for $1 \mathrm{~h}$. After washing with stop/wash buffer, sections were treated with anti-digoxigenin conjugate for $30 \mathrm{~min}$ at room temperature and subsequently developed color in peroxidase substrate. The nuclei were counterstained with $0.5 \%$ methyl green. TUNEL-positive cells/areas were determined by bright field microscopy. For quantification, apoptotic areas were quantified using PROGRES GRYPHAX® software (Jenoptik).

To determine GPX4 and 4HNE expression, kidneys of WT and Epha2 ${ }^{-1-}$ mice were harvested 3 days post infection, and snap frozen in Tissue-Tek® OCT. $10 \mu \mathrm{m}$ kidney sections were fixed with cold acetone, rehydrated in PBS, blocked with BSA, and stained overnight using anti-GPX4 or anti-4HNE (ab125066 and ab46545, respectively). Sections were washed and incubated with anti-rabbit IgG $(\mathrm{H}+\mathrm{L})$ coupled with Alexa Fluor ${ }^{\mathrm{TM}} 488$ (Thermo Fisher Scientific). with Alexa Fluor 568 (Thermo Fisher Scientific). To visualize nuclei, cells were stained with DAPI (Prolong Gold antifade reagent with DAPI). Images of the sections (z-stack) were acquired with a Leica TCS SP8 confocal microscope. To enable comparison of the fluorescence intensities among slide, the same image acquisition settings were used for each experiment.

Determination of serum NGAL and TREM-1. Serum NGAL and TREM-1 were measured at day 3 post-infection. Blood was collected by cardiac puncture from each mouse at the time of sacrifice, 307 and stored at $-80^{\circ} \mathrm{C}$ until use. NGAL and TREM-1 concentrations were determined using DuoSet 308 ELISA Kit (DY1857-05 \& DY1187, R\&D Systems).

309 Cytokine and chemokine measurements in vivo. To determine the whole kidney cytokine and chemokine protein concentrations, the mice were intravenously infected with C. albicans SC5314. 
311 The mice were sacrificed after 3 days post-infection, and their kidney were harvested, weighed

312 and homogenized. The homogenates were cleared by centrifugation and the concentration of

313 inflammatory mediators was measured using the Luminex multiplex bead assay (Invitrogen).

314 Generation of BM chimeric mice. Bone marrow chimeric mice were generated as previously

315 described ${ }^{34}$. Briefly, for BM cell transfers, femurs and tibias from 6- to 8-week-old donor wild-type

316 (Epha2 ${ }^{++} ;$CD45.1, or CD45.2) and Epha2 ${ }^{-/-}$(CD45.2) mice were removed aseptically and BM

317 was flushed using cold PBS supplemented with 2 mM EDTA. Recipient wild-type (CD45.1;

318 B6.SJL-Ptprc ${ }^{a} P e p c^{b} /$ BoyJ) and Epha2 ${ }^{-/}$mice were irradiated with 10 Gy and were reconstituted

3196 hours after irradiation with $2.5 \times 10^{6}$ Epha2 $^{+/+}$CD45.2 (WT $\rightarrow$ WT), Epha2 ${ }^{+/+}$CD45.1 (wild-

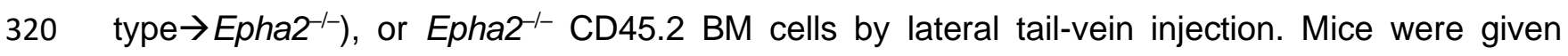

321 enrofloxacin (Victor Medical) in the drinking water for the first 4 weeks of reconstitution before

322 being switched to antibiotic-free water. Chimeras were infected with $C$. albicans 10 weeks after

323 transplantation. Prior to infection, we confirmed that mice reconstituted with congenic BM stem

324 cells had achieved a satisfactory level of chimerism by assessing the number of CD45.1 and

325 CD45.2 leukocytes in the blood, using flow cytometry (Fig. S1).

326 Flow cytometry of infiltrating leukocytes. Immune cells in the mouse kidney were

327 characterized as described. Briefly, mice were infected with C. albicans strain SC5314. After 3

328 days of infection, mice were anesthetized using ketamine/xylazine and perfused with $10 \mathrm{ml}$ of

329 PBS before kidney harvesting. Single-cell suspensions from kidney were prepared using

330 previously described methods ${ }^{101}$. In brief, kidneys were finely minced and digested at $37^{\circ} \mathrm{C}$ in

331 digestion solution (RPMI 1640 with 20 mM HEPES [Gibco] without serum) containing liberase TL

332 (Roche) and grade II DNAse I (Roche) for 20 minutes with shaking. Digested tissue was passed

333 through a 70- $\mu \mathrm{m}$ filter and washed. The remaining red blood cells were lysed with ACK lysis buffer

334 (Lonza). The cells were suspended in 40\% Percoll (GE Healthcare). The suspension was overlaid

335 on $70 \%$ Percoll and centrifuged at $836 \mathrm{~g}$ for 30 minutes at room temperature. The leukocytes and

336 nonhematopoietic cells at the interphase were isolated, washed 3 times in FACS buffer $(0.5 \%$ 
BSA and $0.01 \%$ NaN3 in PBS). The single-cell suspensions were then incubated with rat antimouse CD16/32 (2.4G2; BD Biosciences) for 10 minutes (1:100) in FACS buffer at $4^{\circ} \mathrm{C}$ to block Fc receptors. For staining of surface antigens, cells were incubated with fluorochrome-conjugated (BV421, BV711, FITC, PE, PE-Cy7, allophycocyanin [APC], APC-Cy7) antibodies against mouse CD45 (30-F11; BD Biosciences), Ly6C (AL-21; BD Biosciences), Ly6G (1A8, BioLegend), CD11b (M1/70; eBioscience), CD11c (N41, BioLegend), MHCII (M5/114.15.2, BioLegend), and CD206 (C068C2; BioLegend). After washing with FACS buffer, the cell suspension was stained with a 344 Fixable Viability Stain 510 (BD Biosciences), washed, and resuspended in FACS buffer. The stained cells were analyzed on a FACSymphony system (BD Biosciences), and the data were analyzed using FACS Diva (BD Biosciences) and FlowJo software (Treestar). Only single cells were analyzed, and cell numbers were quantified using PE-conjugated fluorescent counting beads (Spherotech).

RNA Sequencing. Total RNA was isolated as described before ${ }^{102}$. Briefly, kidneys from infected mice were harvested at 3 days post infection andplaced for 1 hour in RNAlater solution negative magnetic bead selection (MojoSort Mouse Pan Dendritic Cell Isolation Kit, BioLegend). $2.5 \times 10^{6}$ BMDCs per well were cultured for $6 \mathrm{~h}$ in 6 -well plates in RPMI complete (R10; $10 \% \mathrm{HI}$ -

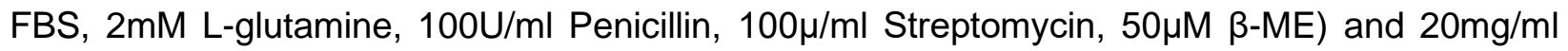
GM-CSF in presence of $25 \mu \mathrm{g} / \mathrm{ml}$ of Curdlan. RNA was isolated using the RNeasy Kit (Qiagen). 357 RNA sequencing was performed by Novogene Corporation Inc. (Sacramento, USA). mRNA was 358 purified from total RNA using poly-T oligo-attached magnetic beads. To generate the cDNA library 359 the first cDNA strand was synthesized using random hexamer primer and M-MuLV Reverse 360 Transcriptase $\left(\right.$ RNase $\left.\mathrm{H}^{-}\right)$. Second strand cDNA synthesis was subsequently performed using 361 DNA Polymerase I and RNase H. Double-stranded cDNA was purified using AMPure XP beads and remaining overhangs of the purified double-stranded cDNA were converted into blunt ends 
via exonuclease/polymerase. After 3 ' end adenylation a NEBNext Adaptor with hairpin loop structure was ligated to prepare for hybridization. In order to select cDNA fragments of 150 200 bp in length, the library fragments were purified with the AMPure XP system (Beckman Coulter, Beverly, USA). Finally, PCR amplification was performed and PCR products were purified using AMPure XP beads. The samples were read on an Illumina NovaSeq 6000 with $\geq 20$ million read pair per sample.

Downstream Data Processing. Downstream analysis was performed using a combination of programs including STAR, HTseq, and Cufflink. Alignments were parsed using Tophat and differential expressions were determined through DESeq2. KEGG enrichment was implemented by the ClusterProfiler. Gene fusion and difference of alternative splicing event were detected by Star-fusion and rMATS. The reference genome of Mus musculus (GRCm38/mm10) and gene model annotation files were downloaded from NCBI/UCSC/Ensembl. Indexes of the reference genome was built using STAR and paired-end clean reads were aligned to the reference genome using STAR (v2.5). HTSeq v0.6.1 was used to count the read numbers mapped of each gene. The FPKM of each gene was calculated based on the length of the gene and reads count mapped to it. FPKM, Reads Per Kilobase of exon model per Million mapped reads, considers the effect of sequencing depth and gene length for the reads count at the same time. Differential expression analysis was performed using the DESeq2 R package (2_1.6.3). The resulting $P$-values were adjusted using the Benjamini and Hochberg's approach for controlling the False Discovery Rate (FDR). Genes with an adjusted $P$-value $<0.05$ found by DESeq2 were assigned as differentially expressed. To allow for log adjustment, genes with 0 FPKM are assigned a value of 0.001 . Correlation were determined using the cor.test function in R with options set alternative = "greater" and method = "Spearman." To identify the correlation between the differences, we clustered different samples using expression level FPKM to see the correlation using hierarchical clustering distance method with the function of heatmap, SOM (Self-organization mapping) and kmeans using silhouette coefficient to adapt the optimal classification with default parameter in $\mathrm{R}$. We 
used clusterProfiler R package to test the statistical enrichment of differential expression genes

in KEGG pathways. The high-throughput sequencing data from this study have been deposited with links to BioProject accession number PRJNA773053 and PRJNA773073 in the NCBI BioProject database.

RNAscope. SCL7a11, Gpx4 and Lyz2mRNA was detected using RNAscope® in situ hybridization. Sections were thawed and postfixed for 15 min in $4 \%$ paraformaldehyde $\left(4 \mathrm{C}^{\circ}\right)$, and washed in PBS at room temperature. In situhybridization was performed using the

396 RNAscope® Multiplex v2 Fluorescent Assay (Advanced Cell Diagnostics, Inc.) in strict 397 accordance with the manufacturer's instruction. Probes used were against 398 mouse Slc7a11 (RNAscope® Probe-Mm-C1, Mm-Slc7a11), Gpx4 (RNAscope® Probe-Mm-C2, 399 Mm-Gpx4-O1 targeting 12-877 of NM_008162.4) and Lyz2 (RNAscope® Probe-Mm-C3, Mm400 Lyz2). The commercially available negative control probe was used, which is designed to target 401 the DapB gene from Bacillus subtilis. In brief, endogenous peroxidases present in the tissue were blocked with an RNAscope® hydrogen peroxidase solution. Tissue was washed in distilled water, then immersed in $100 \%$ ethanol, air dried and a hydrophobic barrier was applied to the slides.

404 The sections were permeabilized with RNAscope ${ }^{\circledR}$ protease III for 30 min at $40^{\circ} \mathrm{C}$. Sections were 405 hybridized with the S/c7a11, Gpx4, and Lyz2 probes at $40^{\circ} \mathrm{C}$ for $2 \mathrm{~h}$. This was followed by a series 406 of amplification incubation steps: Amp 1, $30 \mathrm{~min}$ at $40^{\circ} \mathrm{C}$; Amp 2, $30 \mathrm{~min}$ at $40^{\circ} \mathrm{C}$; $\mathrm{Amp} 3,15 \mathrm{~min}$ 407 at $40^{\circ} \mathrm{C}$. Sections were washed with provided washing buffer $2 \times 2$ min in between each 408 amplification step. Assignment of Akoya 520 (FITC slc7a11), 570 (Cy3 Gpx4) and 690 (Cy5 Lyz2) 409 occurred with a peroxidase blocking step sequentially. Finally, DAPI stain was applied and 410 sections were coverslipped with Invitrogen Prolong Gold antifade mounting medium. Images were 411 taken with the Leica 3D culture Thunder imaging system. For analysis of two representative 412 sections of the renal cortex were taken for each section for two sections (total of $n=4$ per animal). 413 Images were analyzed using Image J. Each channel was thresholded with the Otsu filter circularity 414 set to $0.25-1$ and particle size set to 10-Infinity. Thresholded images were then analyzed and total 
counts were used to represent each gene. Each gene was then normalized to total cell count as assessed by DAPI positive cell count.

Bone marrow isolation and cell differentiation. Bone marrow cells were flushed from femurs and tibias using RPMI 1640 medium (Gibco) supplemented with 10\% HI-FBS and passed through a $70 \mu \mathrm{m}$ cell strainer. Bone marrow derived macrophages were generated by growing freshly isolated bone marrow cells from WT and Epha2 ${ }^{-1-}$ mice in presence of $25 \mathrm{ng} / \mathrm{ml} \mathrm{M-CSF}$ during 7 days in DMEM supplemented with $10 \%$ HI-FBS and Pen/Strep $(100 \mathrm{U} / \mathrm{ml}$ and $100 \mu / \mathrm{ml}$ respectively, Gemini Bioproducts) at $37^{\circ} \mathrm{C}$ in a humidified atmosphere containing $5 \% \mathrm{CO}_{2}$. Bone marrow derived dendritic cells were generated by growing freshly isolated bone marrow cells from WT and Epha2 ${ }^{--}$mice in presence of $20 \mathrm{ng} / \mathrm{ml} \mathrm{GM-CSF}$ during 8 days in RPMI complete (R10) at $37^{\circ} \mathrm{C}$ in a humidified atmosphere containing $5 \% \mathrm{CO}_{2}$. BMDC purity was determined by flow cytometry (>95\%).

Neutrophil killing assay. The capacity of neutrophils to kill $C$. albicans hyphae was determined using the alamarBlue (Invitrogen) reduction as a measure of fungal inactivation. Neutrophils from mice were isolated as described above. Neutrophils were incubated in duplicate wells of flat bottom 96-well plates containing hyphae that had been grown for 3 hours with or without serum opsonization ( $2 \%$ heat-inactivated mouse serum; Gemini Bio-Products), at a neutrophil to $C$. albicans ratio of $1: 4$ at $37^{\circ} \mathrm{C}$. After 2.5 hours, the neutrophils were lysed with $0.02 \%$ Triton X-100 in water for 5 minutes, after which the $C$. albicans hyphae were washed twice with PBS and incubated with $1 \times$ alamarBlue (Invitrogen) for 18 hours at $37^{\circ} \mathrm{C}$. Optical density at a wavelength of $570 \mathrm{~nm}$ and $600 \mathrm{~nm}$ was determined. Neutrophil killing capacity was calculated as the amount of alamarBlue reduced by wells containing $C$. albicans hyphae incubated with and without neutrophils.

Macrophage killing assay. Bone marrow-derived macrophages were generated as described above. Macrophage killing of $C$. albicans yeast was determined by CFU enumeration. Briefly, unopsonized $C$. albicans SC5314 yeast cells were incubated with BMDMs in a 1:1 ratio for 6 and 
44112 hours, respectively. Macrophages were lysed with $0.02 \%$ Triton X-100 in ice-cold water for

4425 minutes, diluted and remaining Candida cells were quantitatively cultured. To determine the

443 effect of ferroptosis inhibition during killing BMDMs were incubated with $10 \mu \mathrm{M}$ Fer-1 or 50ng/ml

444 rmIL-23 for 1 hour prior to infection.

445 Quantification of ferroptosis in vitro. After 7 days of differentiation BMDMs were collected and 446 seeded on fibronectin-coated glass coverslips in presence or absence of $10 \mu \mathrm{M}$ Fer- 1 or $50 \mathrm{ng} / \mathrm{ml}$ 447 rmIL-23, after 1h BMDMs were infected with C. albicans SC5314 at MOI 1. After 210 min Bodipy ${ }^{\mathrm{TM}}$ $448581 / 591 \mathrm{C} 11$ was added at the concentration of $10 \mu \mathrm{M}$ for 30 minutes. Cells were fixed using $2 \%$ 449 paraformaldehyde diluted in PBS. After washing the coverslip with PBS, cells were mounted on 450 microscopic glass using ProLong Gold Antifade Mountant with DAPI. The total fluorescent 451 integrated density of the reduced form of Bodipy 581/591 C11 was quantified using ImageJ (v1.8).

452 To measure individual cellular areas and mean fluorescence, an outline was drawn around each 453 cell, along with several adjacent background readings. Total corrected cellular fluorescence 454 (TCCF) was calculated using the following formula. Integrated density - (area of selected cell $\times$ mean fluorescence of background readings).

457 After 240 min cells were fixed, washed, and permebalized following staining with anti-4HNE 458 (ab46545; Abcam). TCFF was of 4HNE was determined as described above.

459 Macrophage survival in vitro. $1 \times 10^{6}$ BMDMs seeded in 24 well plates were incubated with C. 460 albicans (MOI 1 and 5, respectively) in presence or absence of $10 \mu \mathrm{M}$ Fer-1 or 50ng/ml IL-23. 461 After 4 hours BMDMs were harvested and stained with an antibody against F4/80 (BM8, 462 Biolegend) and propidium iodide (BD Biosciences). The stained cells were analyzed on a 463 FACSymphony system (BD Biosciences).

464 Renal tubular epithelial damage. Primary renal proximal tubule epithelial cells (PCS-400-010, 465 ATCC) in a 24-well plate were loaded with ${ }^{51} \mathrm{Cr}$ overnight. The next day, the cells were incubated 466 with $10 \mu \mathrm{M}$ Fer-1 or diluent, and then infected with C. albicans at a multiplicity of infection of 10. 
471 in triplicate.

472 Cytokine measurement in vitro. BMDMs were stimulated for 6 and 12 hours with C. albicans

473 (MOI 1). RTECs were simulated with C. albicans for 12 hours (MOI 5). BMDCs were stimulated 474 with curdlan (50 $\mathrm{gg} / \mathrm{mL}$; Invivogen) or LPS (1 $\mathrm{gg} / \mathrm{mL}$, Sigma Aldrich) for 24 hours. Supernatant 475 were collected and cytokines were measured with Luminex Bead array (R\&D Systems) or ELISA 476 (TNFa \#DY410, and IL-23 \#D2300B; R\&D Systems). To determine the effect of JAK and PPAR

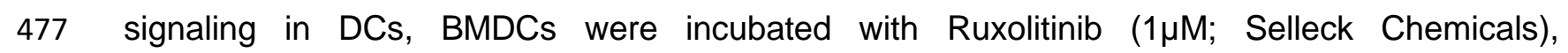
478 Rosiglitazone (10 $\mu \mathrm{M}$; Selleck Chemicals), and GW9662 (10 $\mu \mathrm{M}$; Selleck Chemicals) for 1 hour 479 prior stimulation.

Quantification and statistical analysis. At least three biological replicates were performed for all in vitro experiments unless otherwise indicated. Data were compared by Mann-Whitney corrected for multiple comparisons using GraphPad Prism (v. 9) software. P values $<0.05$ were considered statistically significant.

Data Availability. The authors declare that the data supporting the findings of this study are available within the paper, the accompanying supplementary information files, and the source data (Source Data file).

488 Immunologists Careers in Immunology Fellowship, and a Lundquist Seed grant. MSL is supported 489 by the Division of Intramural Research of the NIAID. RTW is supported by NIH grant 490 R15Al133415, and NJ by the Francis family foundation and by NIH National Center for Advancing 491 Translational Science (NCATS) UCLA CTSI Grant Number KL2TR001882. The content is solely 
492 the responsibility of the authors and does not necessarily represent the official views of the

493 National Institutes of Health.

494 We thank members of the Division of Infectious Diseases at Harbor-UCLA Medical Center

495 for critical suggestions.

496 Author contributions. NM and MS designed the experiments. NM, NVS, DA, NJ, and MS

497 performed the experiments. NM, NVS, DA, NJ, and MS analyzed the data. MSL and RTW

498 provided methodology. MS wrote the manuscript. All authors reviewed and edited. 


\section{Literature}

$5001 . \quad$ Netea, M.G., Joosten, L.A., van der Meer, J.W., Kullberg, B.J. \& van de Veerdonk, F.L.

$501 \quad$ Immune defence against Candida fungal infections. Nat Rev Immunol 15, 630-42 (2015).

$502 \quad 2 . \quad$ Lionakis, M.S., Iliev, I.D. \& Hohl, T.M. Immunity against fungi. JCI Insight 2, 93156

$503 \quad$ (2017).

504 3. Jawale, C.V. \& Biswas, P.S. Local antifungal immunity in the kidney in disseminated

$505 \quad$ candidiasis. Current Opinion in Microbiology 62, 1-7 (2021).

506 4. Brown, G.D. Innate antifungal immunity: the key role of phagocytes. Annu Rev Immunol

$507 \quad 29,1-21(2011)$.

508 5. Dambuza, I.M., Levitz, S.M., Netea, M.G. \& Brown, G.D. Fungal Recognition and Host

509 Defense Mechanisms. Microbiol Spectr 5, 0050-2016 (2017).

510 6. Branzk, N. et al. Neutrophils sense microbe size and selectively release neutrophil

511

512

513

514

515

516

517

518

519

520

521

522

523

524

525

526

527

528

529

530

531

532

533

534 extracellular traps in response to large pathogens. Nat Immunol 15, 1017-25 (2014).

7. Drummond, R.A. et al. CARD9-Dependent Neutrophil Recruitment Protects against Fungal Invasion of the Central Nervous System. PLoS Pathog 11, e1005293 (2015).

8. Whitney, P.G. et al. Syk signaling in dendritic cells orchestrates innate resistance to systemic fungal infection. PLoS Pathog 10, e1004276 (2014).

9. Jawale, C.V. et al. Restoring glucose uptake rescues neutrophil dysfunction and protects against systemic fungal infection in mouse models of kidney disease. Sci Transl Med 12(2020).

10. Conti, H.R. et al. Th17 cells and IL-17 receptor signaling are essential for mucosal host defense against oral candidiasis. J Exp Med 206, 299-311 (2009).

11. Conti, H.R. et al. IL-17 Receptor Signaling in Oral Epithelial Cells Is Critical for Protection against Oropharyngeal Candidiasis. Cell Host Microbe 20, 606-617 (2016).

12. Swidergall, M. \& Filler, S.G. Oropharyngeal Candidiasis: Fungal Invasion and Epithelial Cell Responses. PLoS Pathog 13, e1006056 (2017).

13. Verma, A., Gaffen, S.L. \& Swidergall, M. Innate Immunity to Mucosal Candida Infections. J Fungi (Basel) 3, 60 (2017).

14. Puel, A. et al. Inborn errors of human IL-17 immunity underlie chronic mucocutaneous candidiasis. Curr Opin Allergy Clin Immunol 12, 616-22 (2012).

15. Lionakis, M.S. \& Levitz, S.M. Host Control of Fungal Infections: Lessons from Basic Studies and Human Cohorts. Annu Rev Immunol 36, 157-191 (2018).

16. Lionakis, M.S., Netea, M.G. \& Holland, S.M. Mendelian genetics of human susceptibility to fungal infection. Cold Spring Harb Perspect Med 4(2014).

17. Swidergall, M. et al. Candidalysin Is Required for Neutrophil Recruitment and Virulence During Systemic Candida albicans Infection. J Infect Dis 220, 1477-1488 (2019).

18. Carpino, N., Naseem, S., Frank, D.M. \& Konopka, J.B. Modulating Host Signaling Pathways to Promote Resistance to Infection by Candida albicans. Frontiers in Cellular and Infection Microbiology 7(2017).

19. Lionakis, M.S. et al. Chemokine receptor Ccr1 drives neutrophil-mediated kidney immunopathology and mortality in invasive candidiasis. PLoS Pathog 8, e1002865 (2012).

20. Pappas, P.G., Lionakis, M.S., Arendrup, M.C., Ostrosky-Zeichner, L. \& Kullberg, B.J. Invasive candidiasis. Nat Rev Dis Primers 4, 18026 (2018).

21. Lone, S.A., Wani, M.Y., Fru, P. \& Ahmad, A. Cellular apoptosis and necrosis as therapeutic targets for novel Eugenol Tosylate Congeners against Candida albicans. Scientific Reports 10, 1191 (2020).

22. Bertheloot, D., Latz, E. \& Franklin, B.S. Necroptosis, pyroptosis and apoptosis: an intricate game of cell death. Cellular \& Molecular Immunology 18, 1106-1121 (2021). 
548

549

550

551

552

553

554

23. Jorgensen, I., Rayamajhi, M. \& Miao, E.A. Programmed cell death as a defence against infection. Nature reviews. Immunology 17, 151-164 (2017).

24. Linkermann, A. et al. Regulated cell death in AKI. J Am Soc Nephrol 25, 2689-701 (2014).

25. Tang, D., Kang, R., Berghe, T.V., Vandenabeele, P. \& Kroemer, G. The molecular machinery of regulated cell death. Cell Research 29, 347-364 (2019).

26. Kim, E.H., Wong, S.W. \& Martinez, J. Programmed Necrosis and Disease:We interrupt your regular programming to bring you necroinflammation. Cell Death Differ 26, 25-40 (2019).

27. Dhuriya, Y.K. \& Sharma, D. Necroptosis: a regulated inflammatory mode of cell death. Journal of Neuroinflammation 15, 199 (2018).

28. Chen, X., Kang, R., Kroemer, G. \& Tang, D. Ferroptosis in infection, inflammation, and immunity. J Exp Med 218(2021).

29. Miao, E.A. et al. Caspase-1-induced pyroptosis is an innate immune effector mechanism against intracellular bacteria. Nature immunology 11, 1136-1142 (2010).

30. Wellington, M., Koselny, K., Sutterwala, F.S. \& Krysan, D.J. Candida albicans triggers NLRP3-mediated pyroptosis in macrophages. Eukaryot Cell 13, 329-40 (2014).

31. Li, T. et al. TSC1 Suppresses Macrophage Necroptosis for the Control of Infection by Fungal Pathogen $<$ em $>$ Candida albicans $</$ em $>$. ImmunoHorizons 5, 90-101 (2021).

32. Gross, O. et al. Syk kinase signalling couples to the Nirp3 inflammasome for anti-fungal host defence. Nature 459, 433-436 (2009).

33. Swidergall, M., Solis, N.V., Lionakis, M.S. \& Filler, S.G. EphA2 is an epithelial cell pattern recognition receptor for fungal beta-glucans. Nat Microbio/ 3, 53-61 (2018).

34. Swidergall, M. et al. EphA2 Is a Neutrophil Receptor for Candida albicans that Stimulates Antifungal Activity during Oropharyngeal Infection. Cell Rep 28, 423-433 e5 (2019).

35. Sun, W. et al. Cutting Edge: EPHB2 Is a Coreceptor for Fungal Recognition and Phosphorylation of Syk in the Dectin-1 Signaling Pathway. J Immunol 206, 1419-1423 (2021).

36. Chen, J. et al. TAGAP instructs Th17 differentiation by bridging Dectin activation to EPHB2 signaling in innate antifungal response. Nature Communications 11, 1913 (2020).

37. Aaron, P.A., Jamklang, M., Uhrig, J.P. \& Gelli, A. The blood-brain barrier internalises Cryptococcus neoformans via the EphA2-tyrosine kinase receptor. Cell Microbiol 20(2018).

38. Kottom, T.J., Schaefbauer, K., Carmona, E.M. \& Limper, A.H. EphA2 is a Lung Epithelial Cell Receptor for Pneumocystis $\beta$-glucans. J Infect Dis (2021).

39. Swidergall, M. Candida albicans at Host Barrier Sites: Pattern Recognition Receptors and Beyond. Pathogens 8, 40 (2019).

40. Swidergall, M. et al. Activation of EphA2-EGFR signaling in oral epithelial cells by Candida albicans virulence factors. PLOS Pathogens 17, e1009221 (2021).

41. Phan, Q.T. et al. The Globular C1q Receptor Is Required for Epidermal Growth Factor Receptor Signaling during Candida albicans Infection. mBio 12, e0271621 (2021).

42. Höft, M.A., Hoving, J.C. \& Brown, G.D. Signaling C-Type Lectin Receptors in Antifungal Immunity. Curr Top Microbiol Immunol 429, 63-101 (2020).

43. Brown, G.D. \& Gordon, S. Immune recognition. A new receptor for beta-glucans. Nature 413, 36-7 (2001).

44. Brown, G.D. Dectin-1: a signalling non-TLR pattern-recognition receptor. Nat Rev Immunol 6, 33-43 (2006).

45. de Saint-Vis, B. et al. Human dendritic cells express neuronal Eph receptor tyrosine kinases: role of EphA2 in regulating adhesion to fibronectin. Blood 102, 4431-40 (2003). 
599

600

601

602

603

604

605

606

607

608

609

610

611

612

613

614

615

616

617

618

619

620

621

622

623

624

625

626

627

628

629

630

631

632

633

634

635

636

637

638

639

640

641

642

643

644

645

646

647

648

46. Finney, A.C. et al. EphA2 Expression Regulates Inflammation and Fibroproliferative Remodeling in Atherosclerosis. Circulation 136, 566-582 (2017).

47. Navarathna, D.H., Roberts, D.D., Munasinghe, J. \& Lizak, M.J. Imaging Candida Infections in the Host. Methods Mol Biol 1356, 69-78 (2016).

48. Dunker, C. et al. Rapid proliferation due to better metabolic adaptation results in full virulence of a filament-deficient Candida albicans strain. Nat Commun 12, 3899 (2021).

49. Lionakis, M.S., Lim, J.K., Lee, C.C. \& Murphy, P.M. Organ-specific innate immune responses in a mouse model of invasive candidiasis. J Innate Immun 3, 180-99 (2011).

50. Norice, C.T., Smith, F.J., Jr., Solis, N., Filler, S.G. \& Mitchell, A.P. Requirement for Candida albicans Sun41 in biofilm formation and virulence. Eukaryot Cell 6, 2046-55 (2007).

51. Liu, Y., Mittal, R., Solis, N.V., Prasadarao, N.V. \& Filler, S.G. Mechanisms of Candida albicans trafficking to the brain. PLoS Pathog 7, e1002305 (2011).

52. Carvalho, A. et al. Immunity and Tolerance to Fungi in Hematopoietic Transplantation: Principles and Perspectives. Frontiers in Immunology 3(2012).

53. Leavy, O. Macrophages: Early antifungal defence in kidneys. Nat Rev Immunol 14, 6-7 (2014).

54. Jae-Chen, S. et al. Mechanism underlying renal failure caused by pathogenic Candida albicans infection. Biomed Rep 3, 179-182 (2015).

55. Spellberg, B., Ibrahim, A.S., Edwards, J.E., Jr. \& Filler, S.G. Mice with disseminated candidiasis die of progressive sepsis. J Infect Dis 192, 336-43 (2005).

56. Singer, E. et al. Neutrophil gelatinase-associated lipocalin: pathophysiology and clinical applications. Acta Physiol (Oxf) 207, 663-72 (2013).

57. Duggan, S., Leonhardt, I., Hunniger, K. \& Kurzai, O. Host response to Candida albicans bloodstream infection and sepsis. Virulence 6, 316-26 (2015).

58. Su, L., Liu, D., Chai, W., Liu, D. \& Long, Y. Role of sTREM-1 in predicting mortality of infection: a systematic review and meta-analysis. BMJ Open 6, e010314 (2016).

59. Jang, H.R. \& Rabb, H. Immune cells in experimental acute kidney injury. Nature Reviews Nephrology 11, 88-101 (2015).

60. Dimitrova, P., Gyurkovska, V., Shalova, I., Saso, L. \& Ivanovska, N. Inhibition of zymosan-induced kidney dysfunction by tyrphostin AG-490. Journal of inflammation (London, England) 6, 13-13 (2009).

61. Khounlotham, M., Subbian, S., Smith, R., 3rd, Cirillo, S.L. \& Cirillo, J.D. Mycobacterium tuberculosis interferes with the response to infection by inducing the host EphA2 receptor. J Infect Dis 199, 1797-806 (2009).

62. Zhang, Y. et al. mTORC1 couples cyst(e)ine availability with GPX4 protein synthesis and ferroptosis regulation. Nature Communications 12, 1589 (2021).

63. Dixon, S.J. \& Stockwell, B.R. The Hallmarks of Ferroptosis. Annual Review of Cancer Biology 3, 35-54 (2019).

64. Yang, W.S. \& Stockwell, B.R. Ferroptosis: Death by Lipid Peroxidation. Trends in cell biology 26, 165-176 (2016).

65. Dixon, S.J. et al. Ferroptosis: an iron-dependent form of nonapoptotic cell death. Cell 149, 1060-72 (2012).

66. Horwath, M.C. et al. Antifungal Activity of the Lipophilic Antioxidant Ferrostatin-1. Chembiochem 18, 2069-2078 (2017).

67. Nur, S. et al. IL-23 supports host defense against systemic Candida albicans infection by ensuring myeloid cell survival. PLoS Pathog 15, e1008115 (2019).

68. Liu, Q. \& Wang, K. The induction of ferroptosis by impairing STAT3/Nrf2/GPx4 signaling enhances the sensitivity of osteosarcoma cells to cisplatin. Cell Biol Int 43, 1245-1256 (2019). 
649

650

651

652

653

654

655

656

657

658

659

660

661

662

663

664

665

666

667

668

669

670

671

672

673

674

675

676

677

678

679

680

681

682

683

684

685

686

687

688

689

690

691

692

693

694

695

696

697

698

699

69. Brown, C.W., Amante, J.J., Goel, H.L. \& Mercurio, A.M. The a6ß4 integrin promotes resistance to ferroptosis. The Journal of cell biology 216, 4287-4297 (2017).

70. Legrand, A.J., Konstantinou, M., Goode, E.F. \& Meier, P. The Diversification of Cell Death and Immunity: Memento Mori. Mol Cell 76, 232-242 (2019).

71. Li, J. et al. Ferroptosis: past, present and future. Cell Death \& Disease 11, 88 (2020).

72. Tang, D., Chen, X., Kang, R. \& Kroemer, G. Ferroptosis: molecular mechanisms and health implications. Cell Research 31, 107-125 (2021).

73. Medzhitov, R., Schneider, D.S. \& Soares, M.P. Disease tolerance as a defense strategy. Science 335, 936-41 (2012).

74. Doll, S. \& Conrad, M. Iron and ferroptosis: A still ill-defined liaison. IUBMB Life 69, 423434 (2017).

75. Gaschler, M.M. \& Stockwell, B.R. Lipid peroxidation in cell death. Biochemical and biophysical research communications 482, 419-425 (2017).

76. Harry, R.S. et al. Metabolic impact of 4-hydroxynonenal on macrophage-like RAW 264.7 function and activation. Chem Res Toxicol 25, 1643-51 (2012).

77. Strasser, D. et al. Syk kinase-coupled C-type lectin receptors engage protein kinase C- $\delta$ to elicit Card9 adaptor-mediated innate immunity. Immunity 36, 32-42 (2012).

78. Kapralov, A.A. et al. Redox lipid reprogramming commands susceptibility of macrophages and microglia to ferroptotic death. Nature Chemical Biology 16, 278-290 (2020).

79. Sun, T. \& Chi, J.T. Regulation of ferroptosis in cancer cells by YAP/TAZ and Hippo pathways: The therapeutic implications. Genes Dis 8, 241-249 (2021).

80. Jaumouillé, V. \& Waterman, C.M. Physical Constraints and Forces Involved in Phagocytosis. Frontiers in immunology 11, 1097-1097 (2020).

81. Bain, J.M. et al. Immune cells fold and damage fungal hyphae. Proceedings of the National Academy of Sciences 118, e2020484118 (2021).

82. Cheng, S.-C. et al. mTOR- and HIF-1a-mediated aerobic glycolysis as metabolic basis for trained immunity. Science (New York, N.Y.) 345, 1250684-1250684 (2014).

83. Vucetic, M., Daher, B., Cassim, S., Meira, W. \& Pouyssegur, J. Together we stand, apart we fall: how cell-to-cell contact/interplay provides resistance to ferroptosis. Cell Death \& Disease 11, 789 (2020).

84. Khader, S.A. \& Thirunavukkarasu, S. The Tale of IL-12 and IL-23: A Paradigm Shift. The Journal of Immunology 202, 629-630 (2019).

85. Thompson, A. \& Orr, S.J. Emerging IL-12 family cytokines in the fight against fungal infections. Cytokine 111, 398-407 (2018).

86. Kim, H.S. et al. Curdlan activates dendritic cells through dectin-1 and toll-like receptor 4 signaling. Int Immunopharmacol 39, 71-78 (2016).

87. Parham, C. et al. A receptor for the heterodimeric cytokine IL-23 is composed of IL12Rbeta1 and a novel cytokine receptor subunit, IL-23R. J Immunol 168, 5699-708 (2002).

88. Oppmann, B. et al. Novel p19 protein engages IL-12p40 to form a cytokine, IL-23, with biological activities similar as well as distinct from IL-12. Immunity 13, 715-25 (2000).

89. Chen, Y. et al. A study on the risk of fungal infection with tofacitinib (CP-690550), a novel oral agent for rheumatoid arthritis. Scientific Reports 7, 6779 (2017).

90. Tsirigotis, P. et al. Treatment of Experimental Candida Sepsis with a Janus Kinase Inhibitor Controls Inflammation and Prolongs Survival. Antimicrobial agents and chemotherapy 59, 7367-7373 (2015).

91. Yang, X.O. et al. STAT3 regulates cytokine-mediated generation of inflammatory helper T cells. J Biol Chem 282, 9358-9363 (2007).

92. Lee, P.W. et al. IL-23R-activated STAT3/STAT4 is essential for Th1/Th17-mediated CNS autoimmunity. JCl insight 2, e91663 (2017). 
93. Yao, R. et al. Pathogenic effects of inhibition of mTORC1/STAT3 axis facilitates Staphylococcus aureus-induced pyroptosis in human macrophages. Cell Communication and Signaling 18, 187 (2020).

94. Smith, A.D. et al. Autocrine IL6-Mediated Activation of the STAT3-DNMT Axis Silences the TNFa-RIP1 Necroptosis Pathway to Sustain Survival and Accumulation of MyeloidDerived Suppressor Cells. Cancer Res 80, 3145-3156 (2020).

95. Wang, W. et al. CD8+ T cells regulate tumour ferroptosis during cancer immunotherapy. Nature 569, 270-274 (2019).

96. Fadok, V.A. et al. Exposure of phosphatidylserine on the surface of apoptotic lymphocytes triggers specific recognition and removal by macrophages. J Immunol 148, 2207-16 (1992).

97. Klöditz, K. \& Fadeel, B. Three cell deaths and a funeral: macrophage clearance of cells undergoing distinct modes of cell death. Cell Death Discovery 5, 65 (2019).

98. Dennehy, K.M., Willment, J.A., Williams, D.L. \& Brown, G.D. Reciprocal regulation of IL23 and IL-12 following co-activation of Dectin-1 and TLR signaling pathways. Eur $J$ Immunol 39, 1379-86 (2009).

99. Wang, H. et al. Targeting EphA2 suppresses hepatocellular carcinoma initiation and progression by dual inhibition of JAK1/STAT3 and AKT signaling. Cell Rep 34, 108765 (2021).

100. Melillo, J.A. et al. Dendritic Cell (DC)-Specific Targeting Reveals Stat3 as a Negative Regulator of DC Function. The Journal of Immunology 184, 2638-2645 (2010).

101. Lionakis, M.S. et al. CX3CR1-dependent renal macrophage survival promotes Candida control and host survival. J Clin Invest 123, 5035-51 (2013).

102. Millet, N., Solis, N.V. \& Swidergall, M. Mucosal IgA Prevents Commensal Candida albicans Dysbiosis in the Oral Cavity. Front Immunol 11, 555363 (2020). 

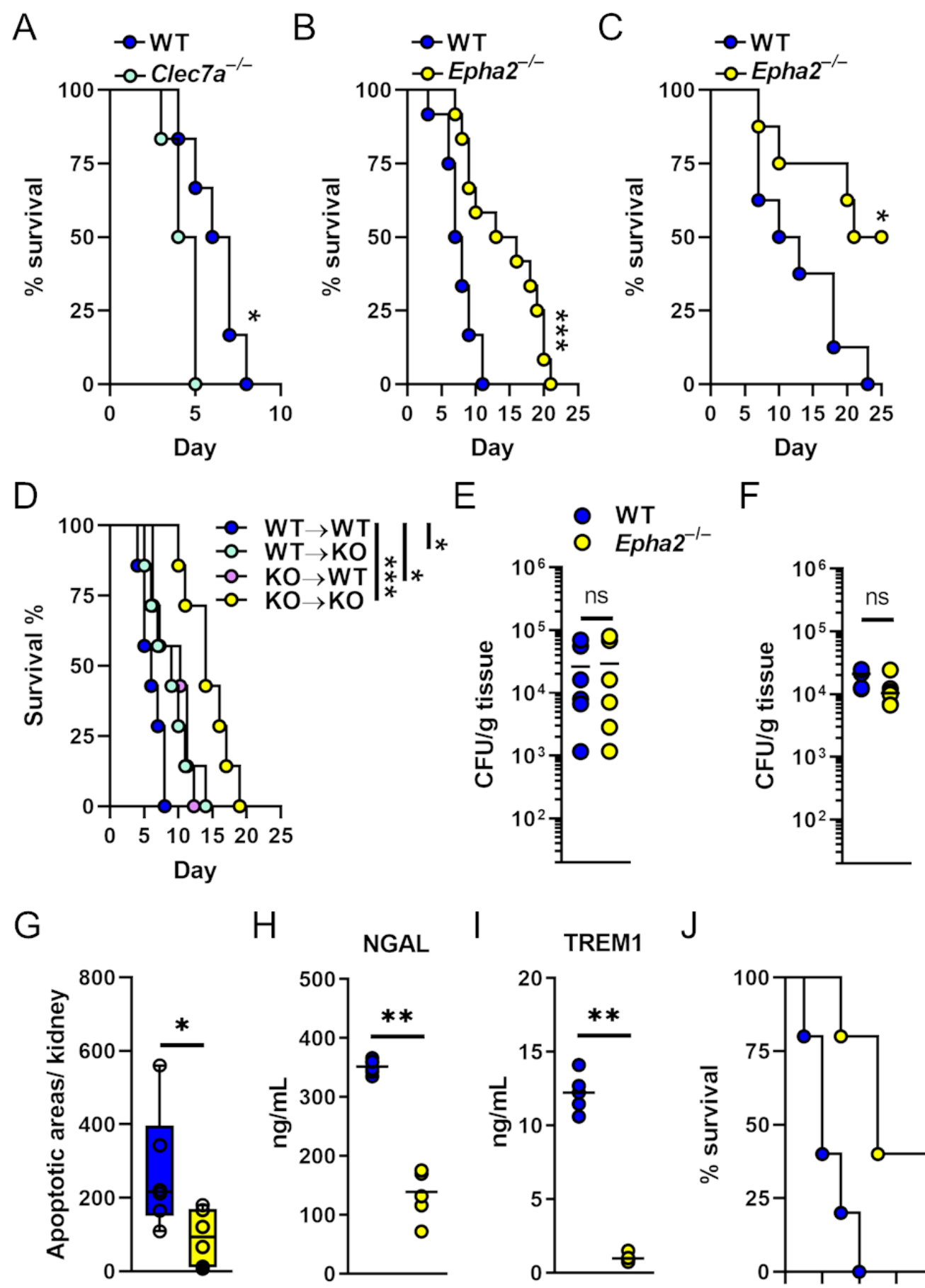

Figure 1. EphA2 promotes disease progression during disseminated candidiasis. A Survival of wild-type and Dectin- $1^{-1-}\left(\mathrm{Clec7a}^{-/}\right)$mice infected intravenously with $2.5 \times 10^{5}$ SC5314 C. albicans. $\mathrm{N}=6$; two independent experiments. B-C Survival of wild-type and Epha2--mice infected intravenously with $2.5 \times 10^{5}$ (B; $N=12$; two independent experiments) or $1.25 \times 10^{5}$ (C; $N=8$; two independent experiments) SC5314 C. albicans. D Survival of bone marrow chimeric mice following infection with $2.5 \times 10^{5}$ SC5314 C. albicans. $\mathrm{N}=7$. Epha2 ${ }^{+/}$mice reconstituted with Epha2 ${ }^{+/+} \mathrm{BM}(\mathrm{WT} \rightarrow \mathrm{WT})$, Epha2 ${ }^{+/+}$mice reconstituted with Epha2 ${ }^{-/} \mathrm{BM}(\mathrm{KO} \rightarrow \mathrm{WT})$, Epha2 
${ }^{-}$mice reconstituted with Epha2 ${ }^{+/} \mathrm{BM}(\mathrm{WT} \rightarrow \mathrm{KO})$, and Epha2 ${ }^{-/} \mathrm{BM}$ to and Epha2 ${ }^{-/}$mice $(\mathrm{KO} \rightarrow \mathrm{KO})$. Statistical significance is indicated by ${ }^{*} P<0.01$, and ${ }^{* * *} P<0,001$. Mantel-Cox Log-Rank test. Kidney fungal burden of infected mice after $\mathbf{E} 4$ days and $\mathbf{F} 12$ hours of infection with $2.5 \times 10^{5}$. Results are median ( $\mathrm{N}=6)$ of two independent experiments. ns, no significance. Mann-Whitney Test. G Apoptotic areas per kidney after 4 days of infection determined by TUNEL staining. $\mathrm{N}=6$; two independent experiments. Statistical significance is indicated by ${ }^{*} P<0.05$; Mann-Whitney Test. $\mathbf{H}$ Serum NGAL and I TREM1 levels after 3 days of infection. $N=6$; two independent experiments. ${ }^{* *} P<0.01$; Mann-Whitney Test. J Survival of wild-type and Epha2 ${ }^{-/}$mice injected intraperitoneally with $750 \mathrm{mg} / \mathrm{kg}$ of zymosan. $\mathrm{N}=5$; two independent experiments. Statistical significance is indicated by ${ }^{* *}, P<0.01$. Mantel-Cox Log-Rank test 
A WT $\bigcirc \mathrm{Epha2}^{-1-}$
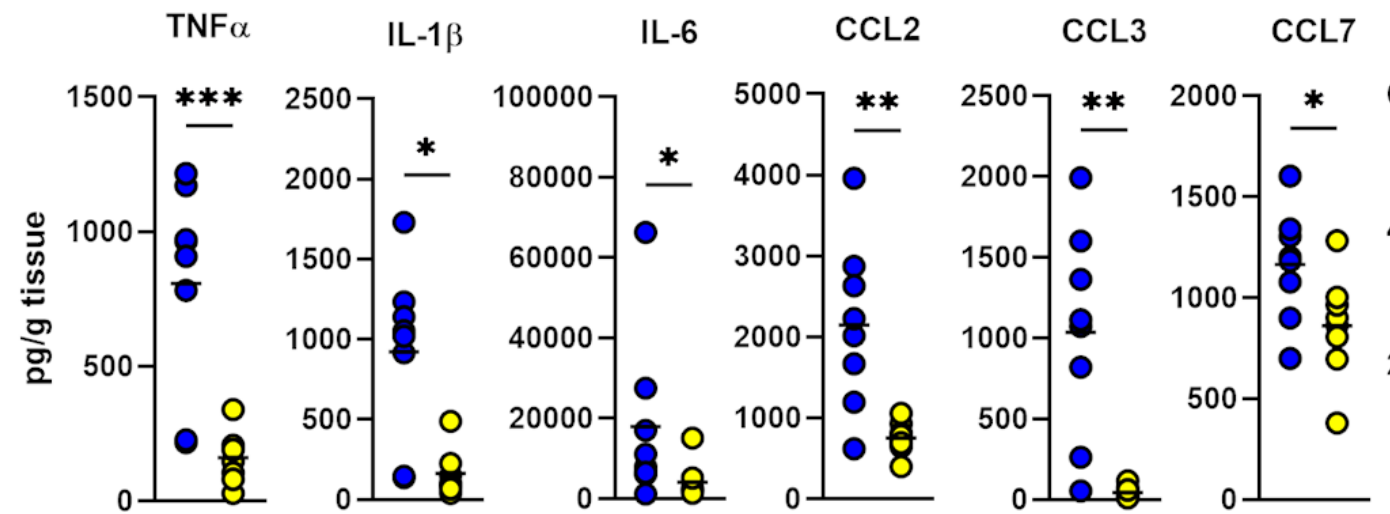

B

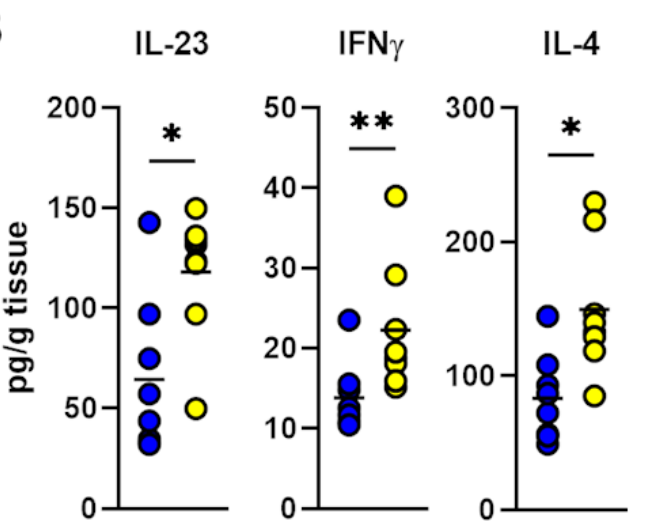

C
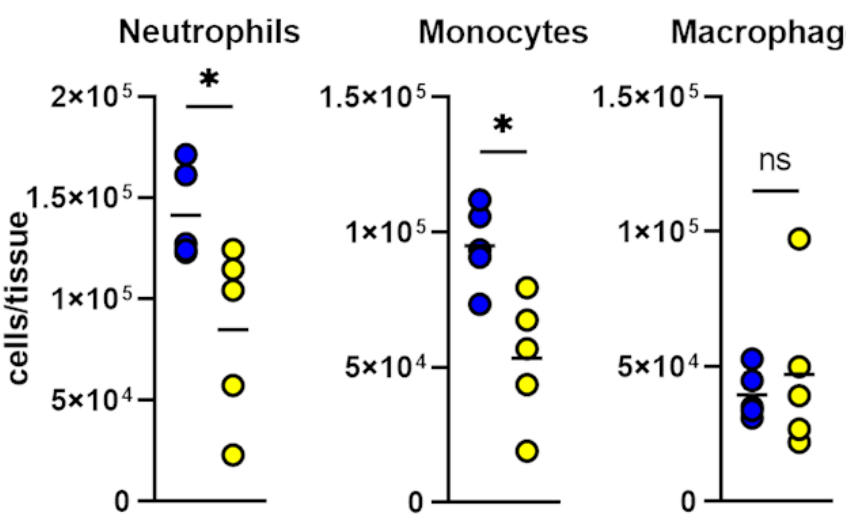

D

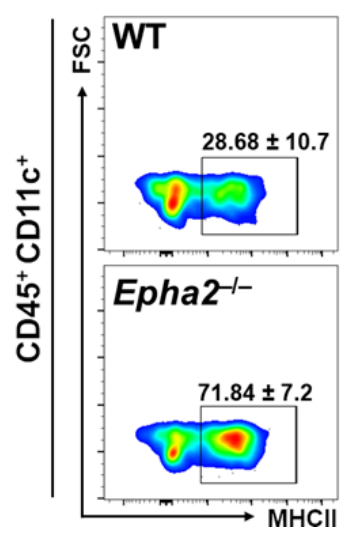

E

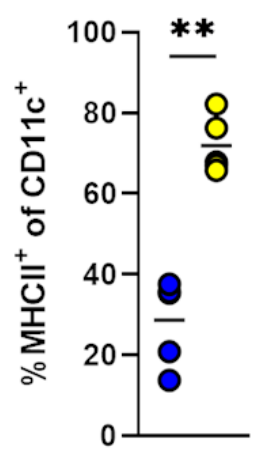

$\mathrm{F}$

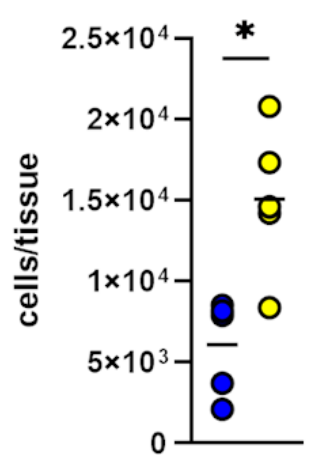

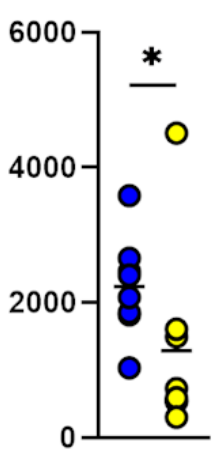

CXCL2
Figure 2. EphA2 deficiency reduces renal neutrophil and monocyte recruitment, but increases DC accumulation during candidiasis. A-B Level of indicated cytokines in kidneys after 3 days of infection. $\mathrm{N}=8$; two independent experiments. Statistical significance is indicated by ${ }^{*} P<0.05$; ${ }^{* *} P<0.01$; ${ }^{* *} P<0.001$. Mann-Whitney Test. $\mathbf{C}$ Accumulation of neutrophils, monocytes, and macrophages in the kidney of wild type and Epha2 ${ }^{-1-}$ mice after 3 days of infection. $\mathrm{N}=5$, two independent experiments. Statistical significance is indicated by; ${ }^{*} P<0.05$. ns; No Significance. Mann-Whitney Test. D-E Representative flow cytometry plots of MHCII expression and frequencies of MHCll-expressing cells in infected kidneys after 3 days of infection. $\mathrm{N}=5$; two experiments. $\mathbf{F}$ Accumulation of conventional dendritic cells (cDCs) and lymphoid dendritic cells (IDCs) in kidneys of wild type and Epha2 ${ }^{-1-}$ mice after 3 days of infection $(\mathrm{N}=5)$. Statistical significance is indicated by ${ }^{*} P<0,05 ;{ }^{*} P<0,01$. Mann-Whitney Test. 
A
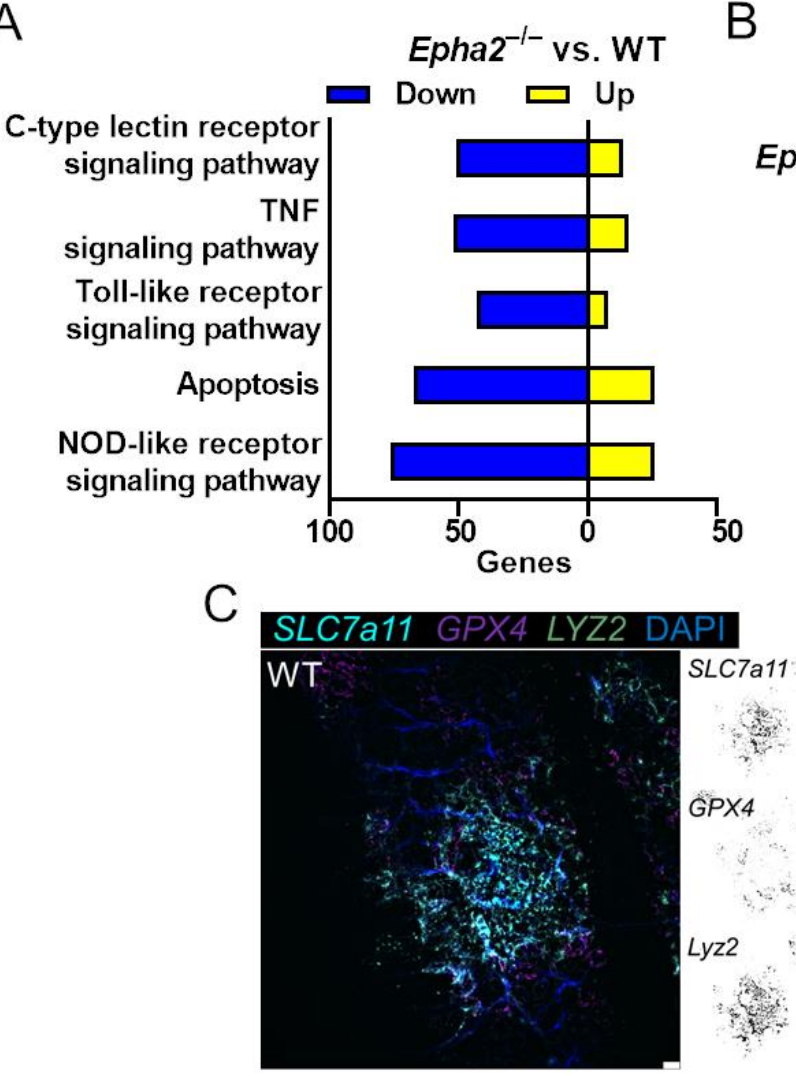

$\mathrm{D}$

norm. RNAscope particles/ $\mathrm{DAPI}^{+}$nuclei

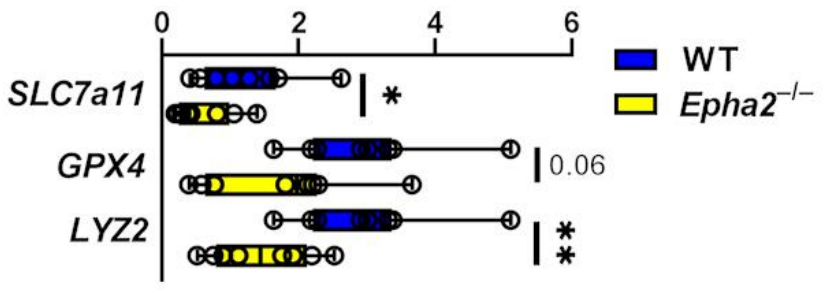

F
$\mathrm{B}$
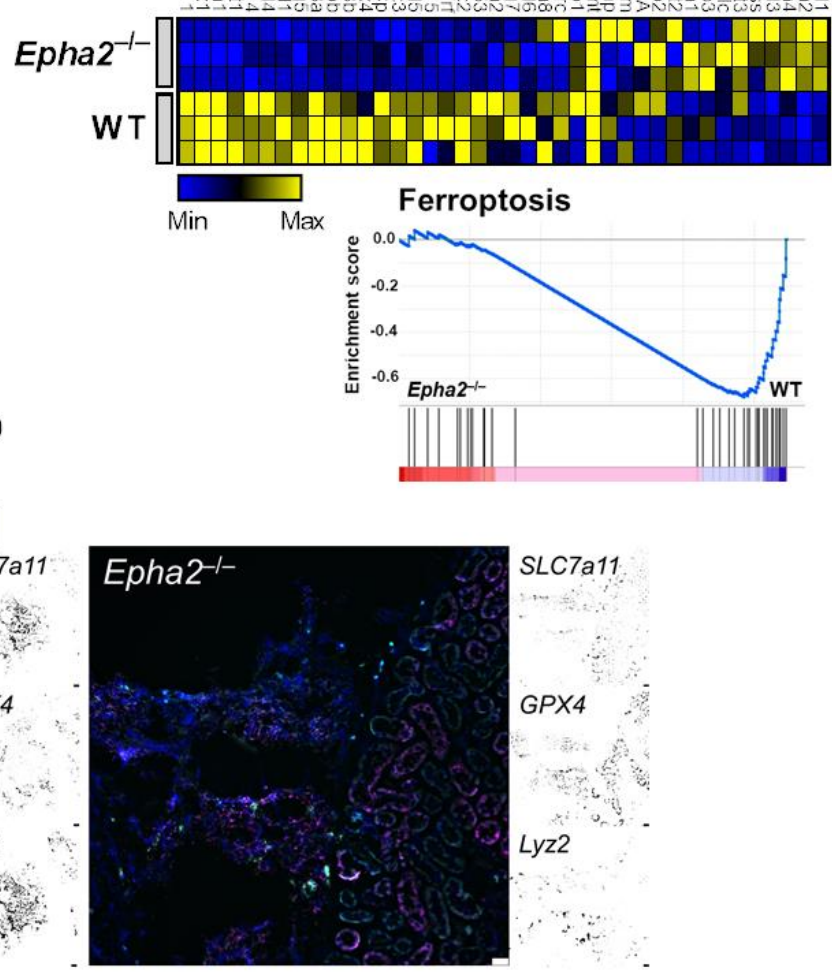

$E$

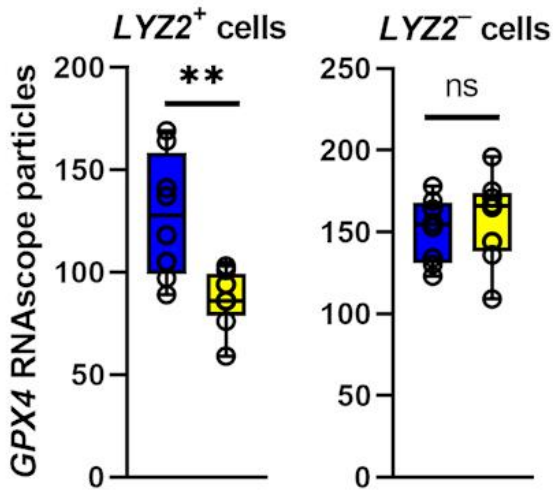

G

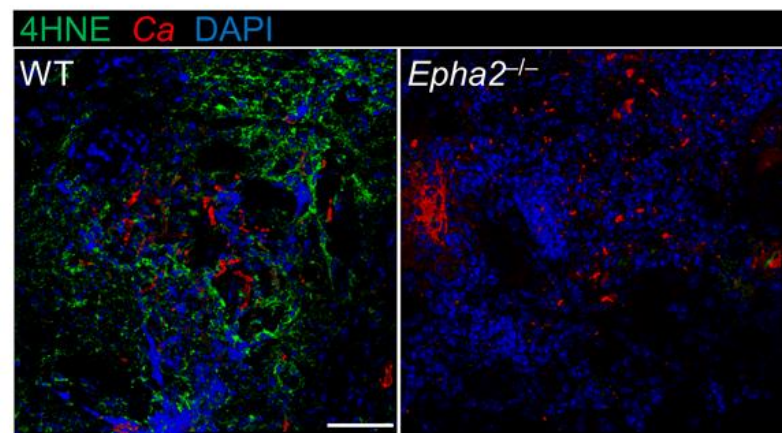

Figure 3. EphA2 promotes ferroptotic host cell death during candidiasis. A Up- and down regulated number of genes of KEGG pathways. RNASeq was performed on mRNA isolated from kidneys of WT and Epha2 ${ }^{-1-}$ mice after 3 days of infection. $\mathrm{N}=3$ per mouse strain. B GSEA of 
763 ferroptosis pathway genes. Heatmap of ferroptosis gene expression analysis in WT and Epha2 ${ }^{-1-}$

764 mice. Normalized enrichment score is shown on Y-axis. C Representative RNAscope image of

765 SCL7a11, GPX4, and LYZ2 expression in infected kidneys after 3 days of infection. Scale bar 50

$766 \mu \mathrm{m}$. D-E Quantification of RNAsope particles. (D) Normalized particles of DAPI+ nuclei. (E) GPX4 ${ }^{+}$

767 particles of $L Y Z 2$ positive and negative cells. $N=4$ per animal. F GPX4 protein expression in

768 infected kidneys after 3 days of infection. GPX4 shown in green, $C$. albicans $(\mathrm{Ca})$ in red. Tissue

769 is visualized using DAPI. Scale bar $100 \mu \mathrm{m}$. G Lipid peroxidation in infected kidneys after 3 days

770 of infection using 4HNE. 4HNE shown in green, $\mathrm{C}$. albicans $(\mathrm{Ca})$ in red. Tissue is visualized using

771 DAPI. Scale bar $100 \mu \mathrm{m}$. 


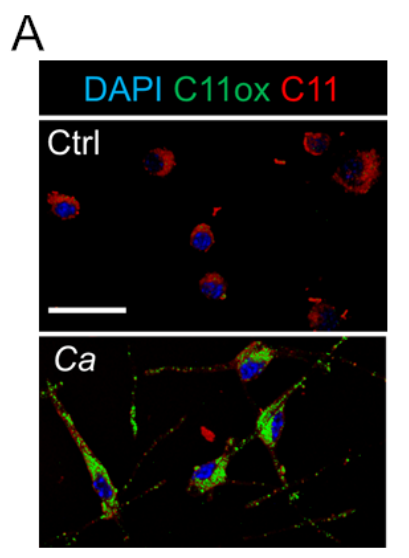

B

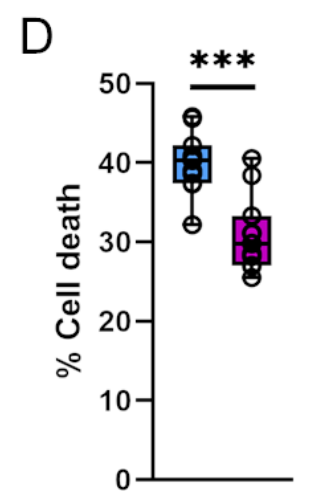

G

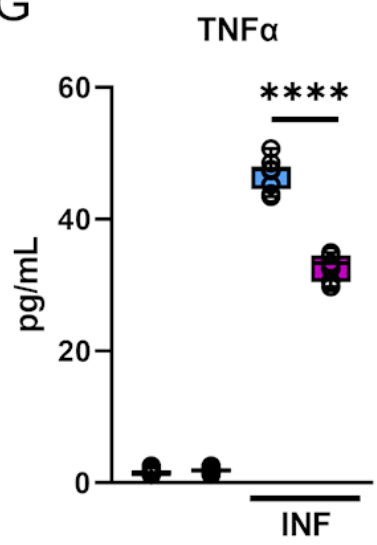

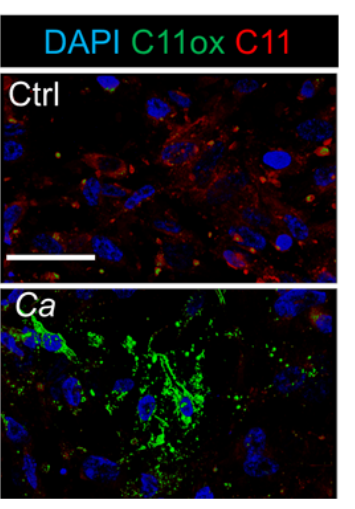

E

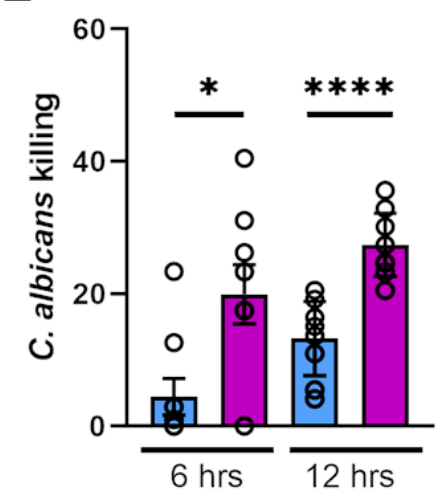

C

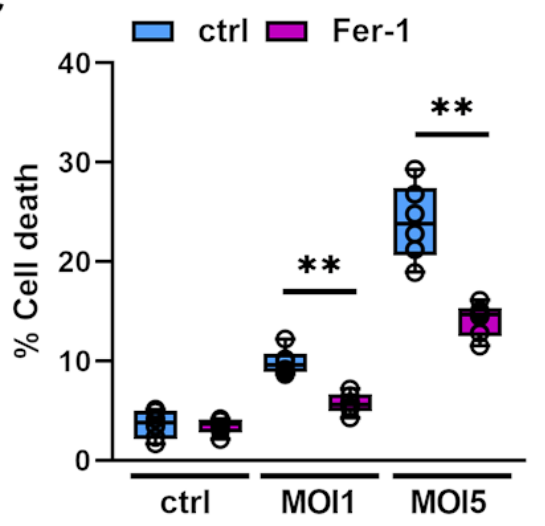

$\mathrm{F}$

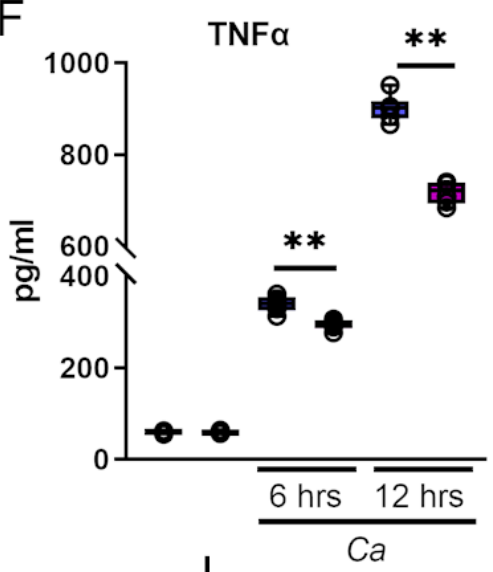

$\mathrm{H}$

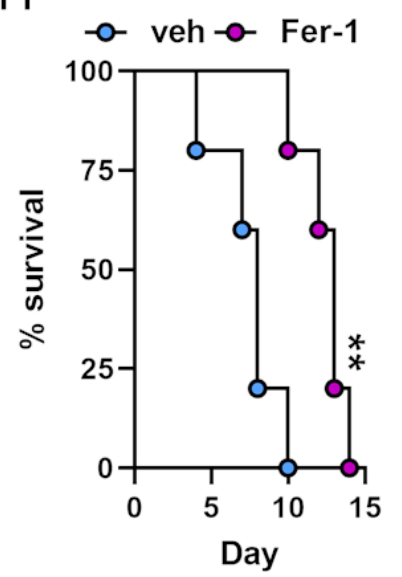

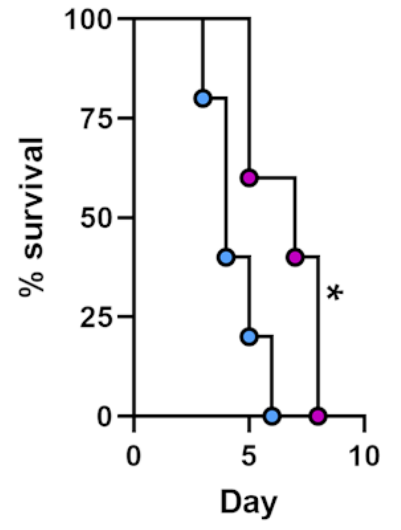

Figure 4. Ferroptotic cell death exerts inflammation, decreases macrophage-mediated fungal killing, and promotes disease progression during candidiasis. A-B Representative images of $C 11$ oxidation of BM-derived macrophages $(A)$ and renal tubular epithelial damage (B) during $C$. albicans infection. Host cells were infected with an MOI of 1 (BMDMs, 4 hours; RTECs, 2 hours). C BM-derived macrophages were treated with $10 \mu \mathrm{M}$ Fer-1 for 1 hour followed by interaction with $C$. albicans (MOI 1 and 5 ) for 4 hours. $\mathrm{PI}^{+}$(dead) cells were determined by gating on $\mathrm{F} 4 / 80^{+}$cells. $\mathrm{N}=3$; duplicate. ${ }^{* *} P<0.01$; Mann-Whitney Test. D Renal tubular epithelial damage determined by specific ${ }^{51} \mathrm{Cr}$ release. $\mathrm{N}=3$; triplicate. ${ }^{* \star}{ }^{*} P<0.001$; Mann-Whitney Test $\mathbf{E} C$. albicans killing of macrophages treated with Fer-1. MOI 1. $\mathrm{N}=3$; triplicate. ${ }^{*} P<0.05$; ${ }^{\star * \star} P<0.001$; MannWhitney Test. F TNFa secretion of BM-derived macrophages during $C$. albicans infection (MOI 1) for indicated time points. $\mathrm{N}=3$, duplicate. ${ }^{* \star} P<0.01$; Mann-Whitney Test. G CXCL8 and TNF $\alpha$ 
bioRxiv preprint doi: https://doi.org/10.1101/2021.12.19.473386; this version posted December 21, 2021. The copyright holder for this preprint (which was not certified by peer review) is the author/funder, who has granted bioRxiv a license to display the preprint in perpetuity. It is made available under aCC-BY-NC-ND 4.0 International license.

784 secretion of RTECs in the presence and absence of Fer-1 during C. albicans infection. MOI 5. $785{ }^{* * *} P<0.0001$; Mann-Whitney Test. H-I Survival of WT mice treated daily with $10 \mathrm{mg} / \mathrm{kg}$ Fer-1 or 786 vehicle control. Inoculum $1.25 \times 10^{5}(\mathrm{H})$ and $2.5 \times 10^{5}$ (I) C. albicans. $\mathrm{N}=5$; two independent 787 experiments per inoculum. ${ }^{*} P<0.05 ;{ }^{* *} P<0.01$. Mantel-Cox Log-Rank test. 
A

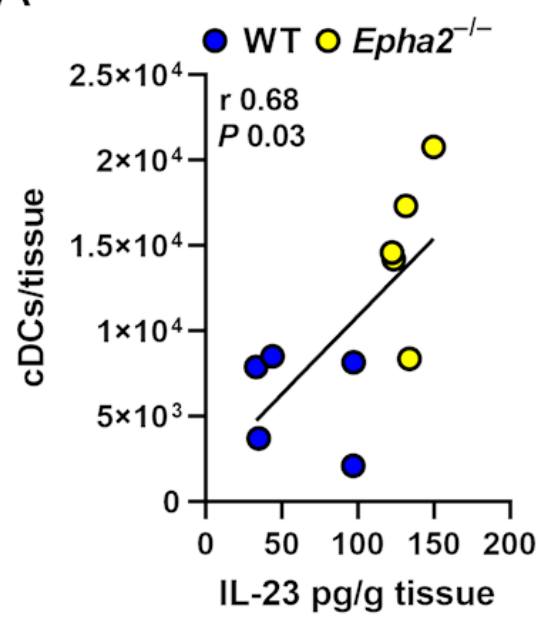

C
B

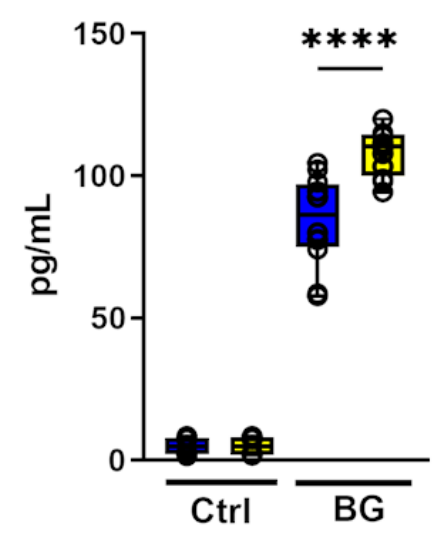

D

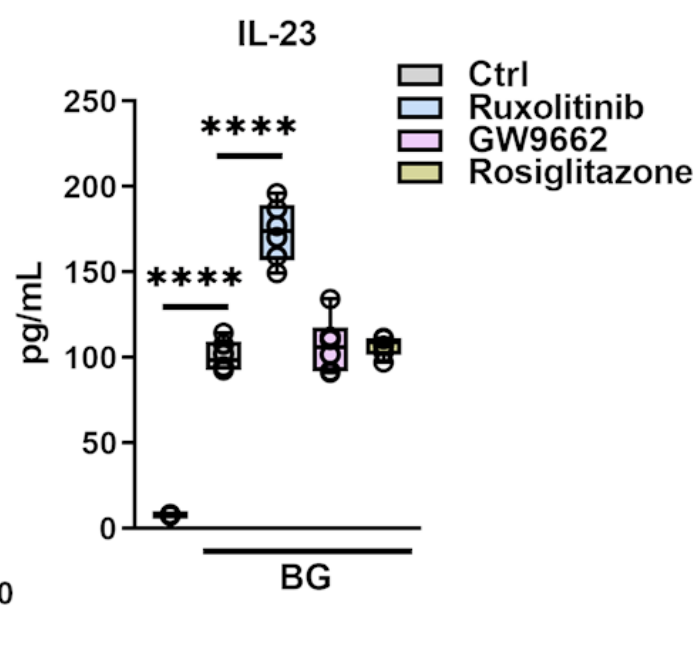

Figure 5. EphA2 signaling decreases IL-23 secretion in BMDCs. A Correlation of IL-23 kidney levels and $\mathrm{CDC}$ infiltration during candidiasis in WT and Epha2 $2^{-1}$ mice. $\mathrm{N}=5$. Pearson correlation coefficient ${ }^{*}, P<0.05$. B IL-23 levels in supernatants of DCs after 24 hours stimulation with $\beta$ glucan (curdlan). DCs generated from WT and Epha2 ${ }^{--}$mice. $\mathrm{N}=6$, duplicate. ${ }^{* \star *}{ }^{*}<0.0001$, Mann-Whitney Test. Ctrl, control; BG, $\beta$-glucan; Ca, C. albicans. C Number of up and down regulated genes in corresponding KEGG pathways. BMDCs from WT and Epha2-/- mice $(n=3)$ were stimulated for 6 hours with $25 \mu \mathrm{g} / \mathrm{mL}$ curdlan. D DC IL-23 secretion treated with indicated inhibitors and stimulated with curdlan. 24 hours post stimulation IL-23 levels in supernatants were measured with ELISA. $\mathrm{N}=3$; duplicate. ${ }^{\star \star \star \star} P<0.0001$, Mann-Whitney Test. 

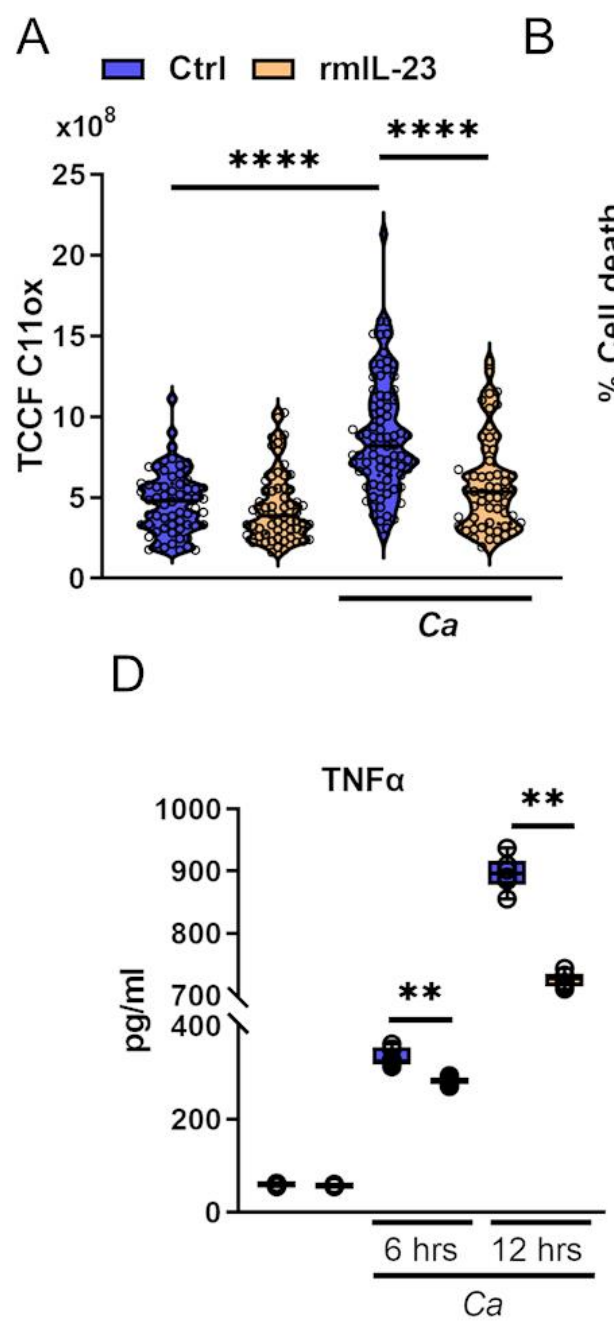

G

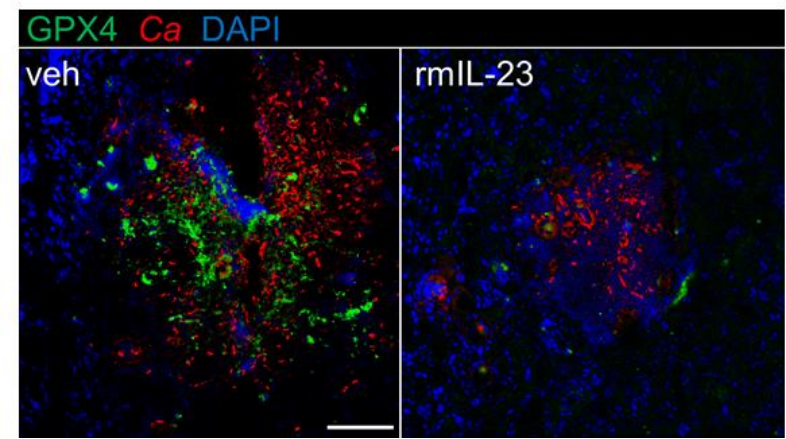

E
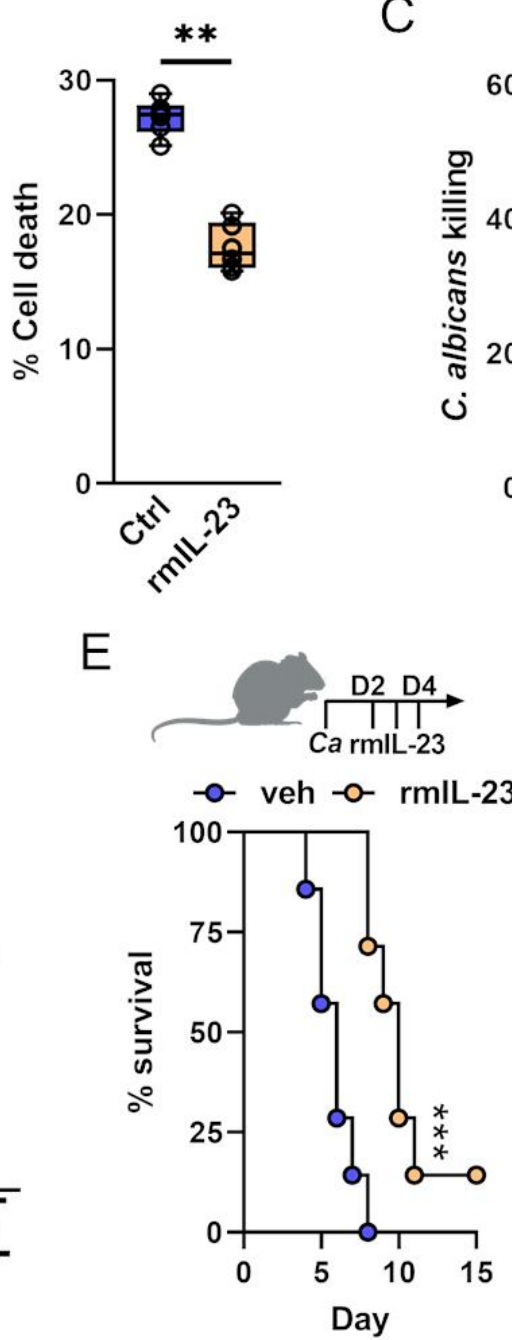

$\mathrm{H}$

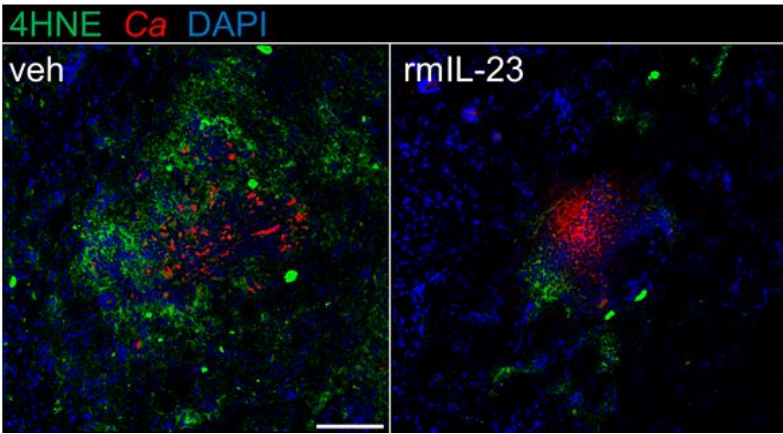

Figure 6. IL-23 signaling reduces ferroptosis during $C$. albicans infection. A BM-derived macrophages were infected with $\mathrm{C}$. albicans (MOI 1) for 4 hours in the presence and absence of rmIL-23. Total C11ox fluorescence was quantified. Individual cells ( $N=57-80 ; 3$ independent experiments) were identified and total fluorescence was measured using ImageJ. ${ }^{* * *} P<0.0001$, Mann-Whitney Test. B BM-derived macrophages were treated with rmlL-23 for 1 hour followed by interaction with $C$. albicans (MOI 5 ) for 4 hours. $\mathrm{PI}^{+}$(dead) cells were determined by gating on F4/80 ${ }^{+}$cells. $\mathrm{N}=3$; duplicate. ${ }^{* *} P<0.01$; Mann-Whitney Test. C Macrophages-mediated $C$. albicans killing in the presence and absence of rmIL-23. MOI 1. N=3; triplicate. ${ }^{*} P<0.05$; ${ }^{* *} P<0.001$; Mann- 
809 Whitney Test D TNFa secretion of BM-derived macrophages during C. albicans infection (MOI 1) 810 for indicated time points. $\mathrm{N}=3$, duplicate. ${ }^{* *} P<0.01$; Mann-Whitney Test. E (Top) IL-23 treatment 811 scheme during disseminated candidiasis. (Bottom) Survival of wild-type mice infected 812 intravenously with $2.5 \times 10^{5}$ SC5314 C. albicans. $\mathrm{N}=7$; two independent experiments. Mice were 813 treated with recombinant murine IL-23 ( $\mathrm{rmlL}-23)$ or PBS. Statistical significance is indicated by $81{ }^{* * *}, P<0.001$. Mantel-Cox Log-Rank test. F Renal fungal burden after 3 days of infection. Single 815 rmlL-23 treatment at day 2. $\mathrm{N}=6-7$; two independent experiments. Mice were treated with 816 recombinant murine IL-23 (rmIL-23) or vehicle (veh; PBS). ${ }^{* *} P<0.01$, Mann-Whitney Test. G 817 GPX4 protein expression in infected kidneys after 3 days of infection. Single rmIL-23 treatment at 818 day 2 relative to infection. GPX4 shown in green, $\mathrm{C}$. albicans $(\mathrm{Ca})$ in red. Tissue is visualized 819 using DAPI. Scale bar $100 \mu \mathrm{m}$. H Lipid peroxidation in infected kidneys after 3 days of infection 820 using 4HNE. Single rmIL-23 treatment at day 2 relative to infection. 4HNE shown in green, $C$. albicans $(\mathrm{Ca})$ in red. Tissue is visualized using DAPI. Scale bar $100 \mu \mathrm{m}$. 